\title{
Combination Strategies for Targeted Delivery of Nanoparticles for Cancer Therapy
}

Zhonglie He

Technological University Dublin

Kangze Liu

Technological University Dublin

Hugh J. Byrne

Technological University Dublin, hugh.byrne@tudublin.ie

See next page for additional authors

Follow this and additional works at: https://arrow.tudublin.ie/nanolbk

Part of the Nanomedicine Commons

\section{Recommended Citation}

$\mathrm{He}$ Z Z. et al(2019). Combination strategies for targeted delivery of nanoparticles for cancer therapy. In (Mohapatra, S, Ranjan, S., Dasgupta, D, Mishra, R., Thomas, S. (eds)) Applications of targeted nano drugs and delivery systems: nanoscience and nanotechnology in drug delivery, Elsevier, pp. 191-219. isbn:9780128140291

This Book Chapter is brought to you for free and open access by the NanoLab at ARROW@TU Dublin. It has been accepted for inclusion in Books / book chapters by an authorized administrator of ARROW@TU Dublin. For more information, please contact arrow.admin@tudublin.ie, aisling.coyne@tudublin.ie, gerard.connolly@tudublin.ie.

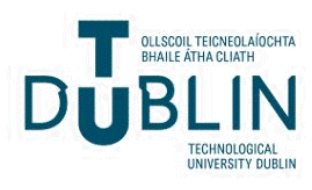




\section{Authors}

Zhonglie He, Kangze Liu, Hugh J. Byrne, Patrick Cullen, Furong Tian, and James Curtin 
Combination Strategies for Targeted Delivery of Nanoparticles for Cancer Therapy Zhonglei He ${ }^{1,2}$, Kangze Liu ${ }^{1}$, Hugh J. Byrne ${ }^{2}$, Patrick J. Cullen ${ }^{1,4}$, Furong Tian ${ }^{* 1,2,3}$, and James F. Curtin ${ }^{* 1,2,3}$

${ }^{1}$ BioPlasma Research Group, School of Food Science and Environmental Health, Dublin Institute of Technology, Dublin, Ireland; ${ }^{2}$ Nanolab, FOCAS Research Institute, Dublin Institute of Technology, Dublin 8, Ireland; ${ }^{3}$ Environmental, Sustainability and Health Research Institute, Dublin Institute of Technology, Dublin, Ireland; ${ }^{4}$ School of Chemical Engineering, UNSW, Sydney, Australia

*Correspondence:

James F Curtin; E-mail: james.curtin@ dit.ie and

Furong Tian; E-mail: furong.tian@ dit.ie

\begin{abstract}
Pharmaceuticals, and more recently biopharmaceuticals, have become the mainstay for antineoplastic treatments in combination with surgical interventions and radiation therapy. In recent years, advances have been made in the development of nanotechnological interventions for the treatment of cancer alone or in combination with existing therapeutic modalities. Nanotechnology used for therapeutic drug delivery and sensitization of photodynamic, sonodynamic and radiotherapy are now being tested in preclinical and clinical trials for the treatment of cancer. This article will review the current state of the art for nanotechnology therapies with an emphasis on targeted drug delivery and the observed and likely benefits when used in combination with existing therapeutic approaches.
\end{abstract}

Key world: Nanoparticles; Nanotechnology; Targeted delivery; Photodynamic therapy; Sonodynamic therapy; Radiotherapy; Sensitization. 


\section{Introduction}

Cancer treatment can be affected by a combination of physical, chemical and biological technologies. Due to the considerable variation between types and status of tumours and individual patients, the efficiency of cancer therapy is difficult to guarantee and commonly associated side effects and off-target toxicity can be daunting $(\mathrm{H}$. Chen et al. 2017). However, recent technological advances have led to the development of new nanotechnological approaches for cancer therapies, which promise high-precision ways to beat cancer. Nanotechnology can be combined with chemotherapy to facilitate targeted delivery into cancer tissue with high specificity and efficacy (Ferrari 2005). Nanoplatforms allow more accurate, non-invasive and real-time cancer diagnosis and monitoring during therapy using magnetic resonance imaging, ultrasonography, etc. (Alexis et al. 2008; Baetke et al. 2015; Zaimy et al. 2017) Adjuvant nanotechnological devices are used in cancer interventions such as radiotherapy (Wu et al. 2016), photodynamic therapy (Clement et al. 2017) and sonodynamic therapy (Xu et al. 2016), which are capable of achieving considerably higher precision of treatment and reduced side effects. After decades of developing the understanding of nanotechnology, cancerrelated nano-treatments have undergone extensive preclinical and clinical-trial studies and shown promising results (H. Chen et al. 2017). Bregoli et al. have summarised of the current state of the art of nanomedicines undergoing clinical trial and clinicallyapproved nanomedicines for cancer therapies (Bregoli et al. 2016).

The traditional definition of a nanotechnological device for cancer treatment is that the essential components of the device or the device itself are artificial, and have at least one dimension in the 1-100 nm range (Ferrari 2005; Whitesides 2003). It has been suggested that there should be less emphasis on the exact definition of size which could be extended to a range of $1-1,000 \mathrm{~nm}$, and define the approaches to bionanotechnology according to their function and purpose (Ferrari 2005). According this definition, nanotechnologies used in cancer treatment include drug-delivery, therapeutic nanovectors, nano-sensitizers, diagnostic agents and macroscopic devices with essential nanocomponents such as microarrays/ 'nanoarrays', nanocantilever arrays and silicon nanowires for highly-specific and highly-efficient molecular detection and diagnostic, etc. (Ferrari 2005) The recent advances and use of these emerging nanotechnological treatments for cancer is briefly reviewed in this chapter with a particular emphasis on nanotechnologies used for drug-delivery and sensitization of therapeutic interventions.

\section{Drug-delivery and Therapeutic Nanovectors}

The applications of nanoparticles in pharmacology have been investigated over the last three decades and a new generation of vehicles for delivery of biomedical compounds has emerged (Couvreur \& Vauthier 2006). Nano-scale carriers have been exploited for drug delivery, drug targeting, histological engineering, tissue targeting and labelling (Kong et al. 2011). The evolution of nanotechnology enables researchers to synthesize a wide variety of nanoparticles with distinct functions and characteristics. Such nanomaterials can be functionalised with molecular and imaging probes or bioactive compounds which can be conjugated, linked, coated or adsorbed to them, in order to implement specific functions (Kong et al. 2011).

For cancer therapy, nanoparticles have been demonstrated to provide site-specific 
delivery by incorporation of various targeted ligands to bind to the desired site, or utilizing stimuli-responsive strategies. Targeted ligands can include various compounds (e.g. antibodies, peptides, hormones, receptor ligands, nucleic acids and lipid derivatives) (Eckmann et al. 2014). Functionalized nanoparticles are capable of responding to one or more physical, chemical, biochemical or environmental stimuli, including osmotic pressure, hydrodynamic pressure, vapor pressure, mechanical force, magnetics, sonophoresis, iontophoresis, hydration, electricity, $\mathrm{pH}$, salt concentration, hydrolysis, enzyme, temperature, light and hypoxia, etc. (Bennet \& Kim 2014) In addition, nanocarriers can prolong circulation time and are incapable of diffusing across non-fenestrated endothelium, thus enabling accumulation in the tumor tissues, resulting in enhanced permeation and retention (EPR). Notably, however, it has been pointed out that precise prognosis should be based on comprehensive characterisation of an individual tumor, rather than broadly on the EPR effect (H. Chen et al. 2017).

The term nanoparticle can encompass a variety of materials, including liposomes, polymer-based nanoparticles, metal-based nanoparticles, dendrimers and combinations of the above. Lipid-based nanoparticles (Liposomes) are the most extensively studied polymeric nanoparticles for biomedical compound delivery (Yatoo 2014). They are membranous lipid bilayer vesicle structures, containing an aqueous volume (Zhao \& Leticia Rodriguez 2012). It has been shown that liposomes and other types of nanocarriers can significantly improve the efficacy of a drug by increasing its solubility, overcoming resistance, controlling the targeted release and modifying their biocompatibility, bioavailability and safety profiles (Tiwle et al. 2012; Fairhurst \& Lee 2012; McAllister et al. 2007; Yatoo 2014; Date et al. 2012). Polymer-based nanoparticles consist of macromolecules which can form a variety of structures (Tian \& Ma 2013; Kroto et al. 1985; Elhissi et al. 2012), which provide specific functions in a variety of fields. Metallic or metal oxide (e.g. gold, iron oxide) nanoparticles have been demonstrated to be useful as imaging and diagnostic agents and have the potential to enhance the performance of various disease diagnostics (Couvreur \& Vauthier 2006). Furthermore, metal-based nanoparticles have been investigated as a specific, promising therapeutic aid to treat cancer in combination with other interventions (Baetke et al. 2015). This section reviews the recent developments of nanoparticles for effective cancer treatment and provides related content that can be used for reference.

\section{Liposomes}

Liposome platforms have attracted considerable attention from the academic and clinical arenas and have become one of the most studied biomaterial nanoparticles, due to the fact that liposomes can significantly improve the efficiency of a drug by increasing its solubility, overcoming resistance, controlling its targeted release and modifying its biocompatibility, bioavailability and safety profile. There have been several clinically approved liposome-based nanomedicines for cancer therapies, such as Doxil, Myocet, Mepact, Dauno Xome, Depocyt, Marqibo and MEPACT (Bregoli et al. 2016). Moreover, advances in liposome research have led to the emergence of hundreds, even thousands, of different functional liposomes for various tumours, which have been tested in preclinical research and clinical trials (Ferrari 2005; Bregoli et al. 2016). According to their number of bilayer membranes, liposomes are grouped into unilamellar and multilamellar vesicles (Zununi Vahed et al. 2017). Unilamellar vesicles have a single lipid bilayer, while multilamellar vesicles consist of several unilamellar 
vesicles surrounded by lipid bilayers. On the another hand, according to the formulation, liposomes can be grouped into several types, including PEGylated stealth liposomes (Couvreur \& Vauthier 2006), immunoliposomes (Tila et al. 2015), lipoplexes (Lonez et al. 2008), fusogenic liposomes (Yuba et al. 2010), stimuli-responsive liposomes (Zununi Vahed et al. 2017) and combinations of the above. (Couvreur \& Vauthier 2006)

Nanoparticles, including liposomes, can be rapidly cleared from the bloodstream by the mononuclear phagocytic system (MPS), significantly decreasing in-vivo circulation half-life and the delivery efficiency of drugs (Bregoli et al. 2016). Therefore, for a longer circulation time, it is critical to protect liposomes from MPS detection. Doxil, the first clinical approved nanomedicine for cancer therapy, is made of polyethylene glycol-coated (PEGylated) liposomes containing anti-cancer drug doxorubicin (Bregoli et al. 2016). Coating liposomes with polyethylene glycol, which is a class of biocompatible, inert and hydrophilic polymer, results in significant increases of the circulation half-life, from several hours to around $45 \mathrm{~h}$, achieving sustained and prolonged drug delivery, and promoting tumour accumulation of liposomes (Couvreur \& Vauthier 2006). PEGylated stealth liposomes have been widely applied in the clinic and are easily functionalised with other functional features, such as stimuli-responses and ligand targeting (Tila et al. 2015). Alternatively, sustained release of a drug from MPS cells, more complex liposomal formulations (e.g. small size, net neutral charge, incorporation of cholesterols and lipids), or drug release into specific areas, such as the cerebrospinal fluid, non-PEGylated liposomes have been demonstrated to result in an increase in the tumour exposure to the drug (Bregoli et al. 2016).

Attaching targeting ligands to the liposome surface is now a well-established and widely used feature in liposome design. Liposomes functionalised with antibodies, also known as immunoliposomes, can carry a drug dose and selectively bind to a chosen tumour site, whilst antibody fragments, glycoproteins, peptides, vitamins and oligonucleotide aptamers can also be used as targeting ligands (Tila et al. 2015; Gunawan \& Auguste 2010; Park et al. 1997; Mastrobattista et al. 1999; Zalipsky et al. 1996). Gene therapies have been considered as a novel and promising method for cancer treatment. Lipoplexes (cationic liposomes) have been investigated as an attractive gene delivery system that are simple to synthesize and control, have high delivery efficiency and can enhance the stability of nucleic acid therapeutics (Tila et al. 2015). Cationic lipids used in lipoplexes are comprised of a cationic head and hydrophobic domain and have the capacity to form particulate complexes in the liposomal membrane by interacting with nucleic acids therapeutics (negatively charged), including plasmid DNA (pDNA), small interfering RNA (siRNA) or microRNA (Tila et al. 2015).

Fusogenic liposomes are able to fuse with cellular membranes and directly release encapsulated drugs into the cytoplasm or targeted cell organelles, significantly enhancing the cellular uptake of drugs, avoiding lysosomal degradation, and counteracting the drug resistance of cancer cells (Tila et al. 2015; Kunisawa et al. 2005). Membrane fusion is achieved by the specific interactions between the membrane receptors and the liposomes or the membrane-associated proteins or peptides that are contained within the liposomes. Moreover, negatively charged phospholipids promote the fusion in the presence of calcium in some types of fusogenic liposomes (Yuba et al. 2010; Malaekeh-Nikouei et al. 2008; Watarai et al. 2014). The formation of lipid bilayers and incorporation of special lipids (e.g. dioleoyl phosphatidylethanolamine (DOPE)) also promote the membrane fusion reaction and enhance the release of the encapsulated drugs (Tila et al. 2015). 
Functional liposomes have been used for the improvement of circulation time and stability of drugs, specifically targeting cancer cells, and promoting drug delivery. More recently, the maturation of stimuli-responsive liposome technologies has yielded precise control of drug release, which provides greater individualised treatment with lower undesirable side effects (Deshpande et al. 2013). Several types of drug release triggers have been used in liposome-based delivery systems, including temperature, $\mathrm{pH}$ level, enzymes, light, ultrasound, electromagnetic waves and magnetic fields. (Zununi Vahed et al. 2017)

The extracellular $\mathrm{pH}$ level in the environment of cancer cells is lower than that around normal cells ( $\mathrm{pH}$ 6.2-6.8 vs $\mathrm{pH}$ 7.1-7.4). Additionally, in endosomal vesicles, the $\mathrm{pH}$ is lower than 5 (Moussa et al. 2015). pH-sensitive liposomes (PSLs) are stable at the neutral $\mathrm{pH}$ of blood and healthy tissues, but are designed to become destabilized and release encapsulated drugs in the vicinity of cancer cells and/or in endosomes (Karanth $\&$ Murthy 2007). The most developed class of PSLs are designed to be triggered after endocytosis and several mechanisms may be involved: direct release of drugs into the cytosol due to the fusion of the endosome and liposome membranes which is induced via $\mathrm{pH}$ changes; drug leakage into the cytosol because of the $\mathrm{pH}$-induced destabilization of liposomal membranes which will cause the destabilization of endosomal membranes; release of drugs inside the endosomes due to the $\mathrm{pH}$-induced destabilisation of the liposomes, followed by the diffusion of the molecules into the cytosol (Moussa et al. 2015). There are several classes of materials used in the formulation of different PSLs, including polymorphic lipids combined with amphiphilic compounds that contain an acidic group, lipid derivatives that have $\mathrm{pH}$-sensitive chemical bond (e.g. $\mathrm{N}$-acylated aminophospholipid derivatives and plasmalogens), reconstituted fusion proteins or peptides that are $\mathrm{pH}$-sensitive and able to destabilise the membrane of liposomes in acidic environments, and $\mathrm{pH}$-titratable polymers, which change conformations at low $\mathrm{pH}$, as recently reviewed by Moussa et al..(Moussa et al. 2015)

Thermo-sensitive liposomes (TSLs) are sensitive to temperature, due to their specific chemical composition. For example, some types of liposomes consist of lipids (e.g. dipalmitoyl phosphatidylcholine) that begin to melt when the temperature exceeds their thermal threshold, whereupon the surface of the liposomes becomes porous and the encapsulated drugs is released (Moussa et al. 2015). The increase of local temperature, known as hyperthermia, can be induced via pathological status or external triggers such as light, ultrasound, microwave or magnetic fields (Tila et al. 2015; Moussa et al. 2015). Optimisation of the hyperthermic effect allows precise control over the amplitude and location of the temperature rise in targeted cancer tissues. Therefore, TSLs which are stable at the temperature of the human body $\left(37^{\circ} \mathrm{C}\right.$ on average), can be triggered to release the encapsulated molecules at targeted sites by hyperthermia (about $39-43^{\circ} \mathrm{C}$ ) to achieve greater therapeutic effect and reduced side effects (Moussa et al. 2015; Ta \& Porter 2013).

Moreover, liposomes can be designed to be sensitive to certain enzymes that only have high activity at the tumour site, such as lipases, cancer-associated proteases and phospholipases(de la Rica et al. 2012; Moussa et al. 2015; Arouri et al. 2013). There are several classes of enzyme-sensitive liposomes, chemically modified with different molecules (e.g. lipopolymers (Arouri et al. 2013), small peptides and phosphorylated synthetic estrogen, etc.(Bibi et al. 2012)) that can specifically respond to certain enzyme levels above a threshold.

Some types of liposomes are capable of absorbing the energy of certain external triggers (e.g. light, ultrasound, electromagnetic waves or magnetic fields, etc.) and subsequently 
converting it to heat, causing a localised hyperthermia that effects the liposomes and induces the release of drugs (Moussa et al. 2015). These external triggers also can be used to directly affect the liposomes. For instance, light can induce changes in the form of photosensitive lipids, which are chemically modified, and change the membrane permeability (Anderson \& Thompson 1992). Ultrasound is able to induce the rupture of microbubbles at target sites, known as transient cavitation, which will be described in detail in section 4 . The collapse of microbubbles produces enormous localised heat and pressure waves, which can cause the disruption of the liposomal and/or cell membranes, consequently allowing the release and permeation of drugs (Hernot \& Klibanov 2008).

Additionally, liposomal platforms which are capable of co-delivering combinatorial drugs bring a paradigm shift in tumour therapy (Hu et al. 2010). Several classes of liposome-based drug combinations have been reported, including the co-delivery of chemotherapeutic drugs, the co-delivery of chemosensitizers and chemotherapies, and the co-delivery of siRNA and chemotherapies (Mayer 2006; Jack Hu \& Zhang 2009; Saad et al. 2008). The combination of drugs can achieve greater synergistic activity by loading them into liposomes at optimised molar ratios and selecting appropriate encapsulation schemes (Hu et al. 2010). For example, multiple hydrophilic drugs can be encapsulated in liposomes; lipophilic drugs can be partitioned into the membrane of liposomes; and negatively charged oligonucleotide drugs (e.g. siRNA) are able to bind to the positively charged liposomal surface (Hu et al. 2010).

\section{Nanogels and polymeric nanoparticles}

Polymeric nanoparticles are nano-sized colloidal particles and have been extensively explored for drug delivery for cancer therapies. Among the various materials, designs and synthesis methods, the polymeric nanoparticles studied most commonly consist of a hydrophobic polymer-based core containing anticancer drugs and a hydrophilic outer shell, which ultimately enabled longer persistence and systemic circulation time in the bloodstram, leading to further accumulation of nanoparticles in cancer tissue (Masood 2016). Alternatively, there has also been increasing interest in using nanosized hydrophilic cross-linked polymer networks, also termed as nanogels, for drug delivery (Chan \& Almutairi 2016; Sivaram et al. 2015).

Polyhydroxyalkanoates (PHAs) (Li \& Loh 2017), cyclodextrins (CDs) (Duchene et al. 2016) and poly-(lactide-co-glycolic acid) (PLGA) (Katiyar et al. 2015) are the most commonly used polymer materials for the core fabrication (Masood 2016). Meanwhile, nontoxic hydrophilic outer shells provide outstanding blood biocompatibility, such as , polyvinyl alcohol (PVA), PEG and monomethoxy poly-(ethylene glycol) (mPEG), which has also been widely applied in surface modification of other kinds of nanoparticles, such as liposomes and gold nanoparticles (Masood 2016). The reader is referred to (Li \& Loh 2017; Duchene et al. 2016; Masood 2016) for an in-depth investigation and discussion of recent advanced PHAs, CDs and PLGA-based polymeric nanoparticles used for cancer treatment.

Nanogels are hydrophilic nanosized cross-linked polymer networks, also called hydrogel nanoparticles (Chan \& Almutairi 2016; Sivaram et al. 2015; Lux et al. 2013). In the last decade, there has been increasing interest in the applications of nanogels as drug carriers and imaging agents (Sivaram et al. 2015; Sultana et al. 2013; Maya et al. 2013). Nanogels have unique utilities and properties including:

1. high biocompatibility on account of the high water content and living tissue- 
like physical properties and they are easily biodistributed by intravenous injection (Soni \& Yadav 2016; Chan \& Almutairi 2016).

2. the ability to selectively respond to stimulation, including changes of $\mathrm{pH}$, ionic content, biomolecules, magnetic field, light and temperature, which is important in specific drug delivery and responsive imaging(Stuart et al. 2010; Eckmann et al. 2014).

3. highly efficient loading capacity of a wide range of drugs due to the prolonged residence time provided by muco-adhesive polymers(Sivaram et al. 2015).

The release of the drugs and other molecules is easy to control by varying the nanogel properties, for example by incorporating stimulus-responsive crosslinkers or changing crosslinking density (Sivaram et al. 2015).

Nanogels are capable of generating appropriately sized complexes with molecules and keeping their configuration and activity, and even encapsulating fragile compounds to increase their stability (Sasaki et al. 2010; Singh et al. 2013; Sivaram et al. 2015; Bae $\mathrm{Ki}$ et al. 2008). Like other nanomaterials, nanogels have nanoscale physical properties, such as size (20 200 nm) and large surface area (Maya et al. 2013; Soni \& Yadav 2016). Meanwhile, the production of nanogels enables versatile formulation and it is facile to chemically modify nanogels for specific purposes, including triggered drug release and targeted drug delivery (Vinogradov et al. 2002; Maya et al. 2013). These properties of nanogels make them promising for applications in anti-skin disease, anti-inflammatory, ocular, transdermal and protein/peptide drug delivery and therapy, cancer drug delivery and imaging (Sivaram et al. 2015).

For cancer therapy, nanogels demonstrate site-specific delivery by incorporating various targeted ligands to bind to a desired site, or utilising the stimuli-responsive ability (Eckmann et al. 2014). Targeting ligands can include various compounds, such as antibodies, peptides, hormones, receptor ligands, nucleic acids and lipid derivatives, etc. In addition, nanogel carriers have prolonged circulation time and are incapable of diffusing across non-fenestrated endothelium, and thus are able to accumulate in tumour tissues (EPR). For instance, Liang et, al. described a novel self-assembled nanogel consisting of hyaluronic acid-epigallocatechin gallate conjugates (HA-EGCG), cytotoxic protein Granzyme B (GzmB) and linear polyethylenimine (PEI) (Liang et al. 2016). HA is known to have the ability of targeting CD44, which is overexpressed in many cancer cell types and EGCG is used to facilitate the formation of stable nanogels. After endocytosis, $\mathrm{PEI}$ is able to change the $\mathrm{pH}$ in endosomes and rupture the membrane to release drugs into the cytosol (Boussif et al. 1995). It has been observed that this nanogel efficiently kills CD44-overexpressing cancer cells and shows little toxic effect to normal cells (Liang et al. 2016). Jicheng Yu et, al. have reported a method to develop a natural particulate-inspired targeted nanogel with endosome membrane components from source cancer cells (EM-NG). EM-NGs have a highly specific homotypic affinity to source cancer cells, but are not ingested by non-source cells (Yu et al. 2016).

After accumulating in tumour tissues via the EPR effect, $\mathrm{pH}$-responsive nanogels are triggered to release drugs either in the extracellular fluids ( $\mathrm{pH} \mathrm{6.8)} \mathrm{or,} \mathrm{after} \mathrm{cellular}$ uptake, in the acidic endosomes and lysosomes ( $\mathrm{pH}$ 4.5-6.5) in cancer cells (Manchun et al. 2015; Manchun et al. 2012). In recent research, hybrid nanogels have been applied to photothermal cancer therapy. Hui Wang et al. fabricated drug loaded core-shell hybrid nanogels that have the function of both tumour imaging, local hyperthermia, 
temperature sensing and triggered drug release (Wang et al. 2014). These multifunctional nanoparticles have high photoluminescence, photostability, magnetic/NIR-heat conversion ability and drug accumulation potential due to the composite structure consisting of fluorescent carbon dots, a porous carbon shell and superparamagnetic iron oxide nanocrystals. Meanwhile, the hydrogel shell can control the release of drug and fluorescence intensity by varying environmental temperature, which is based on the thermo-responsive poly ( $\mathrm{N}$-isopropylacrylamide-co-acrylamide) (Wang et al. 2014). Therefore, NIR light and magnetic field are able to induce localised heating and trigger the release of drugs. In addition, they demonstrated that these hybrid nanogels are capable of overcoming cellular barriers to exert effects in mouse melanoma B16-F10 cells (Wang et al. 2014).

\section{Metal-based nanovectors}

Gold nanoparticles (AuNPs) are the most studied metal-based nanovectors for anticancer drug delivery and have been demonstrated to be promising and effective imaging labels and contrast agents on account of their strong surface plasmon resonance (SPR) effect. Electromagnetic radiation of specific resonant frequencies can induce a coherent oscillation of the free electrons on the nanoparticle surface, when the diameter (1-100 $\mathrm{nm}$ ) of the metal nanoparticles is less than the wavelength of light. The oscillation is called the SPR. The SPR can cause an intensely enhanced absorption and scattering of electromagnetic radiation in resonance with the metal nanoparticles (Jain et al. 2007). Furthermore, AuNPs have high stability and can be easily manufactured in controllable size, shape and functionalised by bioconjugations and biomodifications, which make them a outstanding nanomaterial for drug delivery. It is also known that AuNPs with strong surface-plasmon-enhanced absorption can convert the absorbed light into localized heat expeditiously and therefore can utilised for selective photothermal cancer therapy (El-Sayed et al. 2006).

AuNP are generally considered relatively nontoxic to normal cells (Alkilany \& Murphy 2010; Connor et al. 2005; Villiers et al. 2010; Shukla et al. 2005), but nonfunctionalized AuNPs have been reported to show selective cytotoxicity to certain cell lines, especially cancer cells. Hirak K. Patra and colleagues found that citrate-capped AuNPs (13 nm in diameter) can specifically induce death in the human carcinoma lung cell line (A549), while leaving two other cell lines, baby hamster kidney (BHK21) and human hepatocellular liver carcinoma (HepG2), unaffected at the same dosage (Patra et al. 2007). AuNP have been implicated in detrimental effects on various important cellular components, such as mitochondria and membrane, damage to DNA, generation of reactive chemical species, hindrance of cell function and ultimately induction of cell death (Alkilany \& Murphy 2010). Triphenylphosphine monosulfonate (TPPMS) capped AuNPs (1.4 nm in diameter) were found to be toxic to HeLa cervical carcinoma epithelial cells $\left(\mathrm{IC}_{50}=48 \mu \mathrm{M}\right)$ as a result of oxidative stress and mitochondrial damage leading to necrosis (Pan et al. 2009). Metal nanoparticles can capture electrons from $\mathrm{O}_{2}$, owing to their high surface/volume ratio and the specific electronic configuration of surface atoms (Nel 2007). Jia et Al. reported that AuNPs (13 nm in diameter) can catalyse the production of nitric oxide (NO) from endogenous S-nitroso adducts with thiol groups (RSNOs) in blood serum, resulting in the formation of Au-thiolate on the surface. It is known that NO can effectively interact with superoxide to generate a toxic peroxynitrite (ONOO-) species [ref]. 
Cellular responses to AuNPs depend on their chemical/physical properties, such as surface charge, size and shape (Alkilany \& Murphy 2010). For instance, Goodman and colleagues found that cationic (aminated) AuNP (size $2 \mathrm{~nm}$ ) are able to cause membrane disruption and cell death, while anionic (carboxylated) AuNP with similar size and shape show no toxicity to cells. It suggests that the binding of the AuNPs to the negatively charged cell membrane can be a potential mechanism of the cytotoxicity of cationic AuNP (Goodman et al. 2004).

Recent nanoparticles-cancer research has led to the development of special functional nanosized gold-based particles that conjugate drugs, antibodies or bioactive ligands which are designed to target or kill cancer cell (Ferrari \& Phelps 2005; Cheng et al. 2014; Kong et al. 2011). Branch et al. reported aurimmune CYT-6091, constructed by concurrently binding polyethylene glycol (PEG) and recombinant human Tumour Necrosis Factor (rhTNF) to AuNPs. It was tested in a phase I dose escalation clinical trial. It was demonstrated that the rhTNF plays a role in targeting and killing cancer cells (Libutti et al. 2010). Anil Kumar et al. successfully investigated a kind of peptide functionalized AuNP which consists of a therapeutic peptide, PMI and neuropilin1(Nrp-1) receptor-targeted peptide on ultrasmall AuNPs (2 nm). Since nrp-1 is highly expressed by a variety of human cancer cell lines and PMI is a potent inhibitor of suppressive protein, p53, these AuNPs can target cancer cells and show strong anticancer activity (Kumar et al. 2012).

Metal-based nanoparticles used for drug delivery also include iron oxide (Sharma et al. 2015), zinc oxide (Rasmussen et al. 2010) and titanium oxide (Bakhshizadeh et al. 2017) based nanoparticles. For instance, Sun et al. fabricated iron oxide nanoparticles conjugated with anti-cancer drug methotrexate and targeting ligand chlorotoxin, and enhanced cytotoxicity was observed both in vitro and in vivo (Sun et al. 2008). Meanwhile, due to the superparamagnetic properties of iron oxide nanoparticles, they can also be used as MRI contrast agents for diagnosing and monitoring the effects of tumour treatment at the same time (Sun et al. 2008). To enhance the loading capacity of metal-based nanoparticles, polymer (Bakhshizadeh et al. 2017), lipid (Kong et al. 2012) or other customized shells have been used to coat the metal cores, which also provide high stability and biocompatibility. For instance, Bakhshizadeh et al. assembled core-shell molecularly imprinted polymer for drug delivery by coating titanium oxide nano-cores with diacrylated polycaprolctone as cross-linkers and methacrylic acid or 4-vinylpyridin as the functional modification, and superior loading capacity, higher amount of drug release and considerable cytotoxicity in vitro were observed (Bakhshizadeh et al. 2017).

On the other hand, metal-based nanoparticles, including copper, copper oxide (Studer et al. 2010; Akhtar et al. 2013), titanium oxide (Park et al. 2008), silver (Foldbjerg et al. 2009) have been demonstrated to be able to induce the generation of reactive oxygen species inside cells and ultimately lead to oxidative stress, DNA damage and apoptosis of targeted cells, which provide the therapeutic potential as anti-cancer agents themselves.

\section{Silicon and silica nanoparticles}

As emerging nanomaterials, nanosized porous silicon (silicon-based nanoparticles) and silicon oxide (silica)-based nanoparticles have attracted great attention for cancer treatment applications. Compared to other types of nanoparticles, silicon and silica 
based nanoparticles have uniquely mesoporous structures, strong hydrophobicity and other distinct material characteristics which make them a promising nanomaterial for cancer therapy applications (Feng et al. 2016). Due to the biocompatibility, porous structures/volume and high active surface area of silicon-based nanoparticles, they have been demonstrated to be one of the outstanding candidate nanovectors for targeted drug delivery, diagnosis and sensitisations of sonodynamic, photodynamic and thermal therapies, etc. (Ferrari 2005; Stojanovic et al. 2016) Silicon is one of the most fundamental trace materials in the human body, and silicon-based nanoparticles are completely degradable in the living organism. The generated free silicon atoms can be converted to a nontoxic bioavailable form and be excreted efficiently, which illustrates the biocompatibility of silicon-based nanoparticle (Stojanovic et al. 2016). It was observed using Raman spectroscopy that silicon nanoparticles can be completely biodegraded in breast cancer cells after 13-days incubation (Tolstik et al. 2016). The surface modifications of silicon-based nanoparticles for stabilisation, targeted delivery, controlled drug release or immunotherapy can be accomplished due to the porous structures/volume and high surface area (Stojanovic et al. 2016). Meanwhile, the pores inside silicon-based nanoparticles and their structure allow high-efficiency loading of varied compounds for cancer treatment, including both hydrophilic and hydrophobic compounds (Stojanovic et al. 2016; Kaasalainen et al. 2015; Kinsella et al. 2011; Salonen et al. 2005).

Coating silicon nanoparticles with PEG (Putnam 2008) or serum albumin (Xia et al. 2013) is capable of increasing the solubility and stability of silicon nanoparticles in aqueous environments and results in a much longer circulation time and EPR, similar to other nanoparticles. Simultaneously, silicon nanoparticles can target tumour tissue and enter cancer cells by recognising the receptors on the cell membrane and anchoring via multiple functionalised components, such as carbohydrates, antibodies and peptides (Stojanovic et al. 2016; Secret et al. 2014; Godin et al. 2011). A variety of silicon nanoparticle-delivered drugs have been examined, such as methotrexate, SFN (Wang et al. 2015), indomethacin (Liu et al. 2013), PTX (D. Liu et al. 2015), DOX (Xu et al. 2015) and siRNA (Osminkina et al. 2012), etc.

Silicon oxide, also known as silica, is well-known as a bio-safe and widely-applied natural material. Due to the large internal surface area, controllable porous structure and other material properties of silica-based nanoparticles, they have been investigated as imaging agents, drug vectors and sensitisers as well as silicon-based nanoparticles (Feng et al. 2016). The most commonly studied structures of silica nanoparticles for drug delivery are mesoporous silica based nanoparticles based on Mobil Composition of Matter No. 41 (MCM-41) and/or Santa Barbara Amorphous-15 (SBA-15) (Feng et al. 2016). By altering the temperature, solution composition and concentrations of the synthesis system, the size, shape, surface area and pore size of the hexagonal porous MCM-41 structure can be easily controlled. SBA-15 type silica nanoparticles are synthesized using a polymeric template, which is able to provide mesostructural ordering properties for silica nanoparticles (Zhao 1998). MCM-41 type and SBA-15 type have both been extensively explored for targeted drug delivery (Feng et al. 2016). Controlled-release drug delivery systems based on silica nanoparticles have also attracted increasing attention. The stimuli used for triggered drug release from silica nanoparticles include pH (Casasus et al. 2004), temperature (Yang et al. 2014), redox 
potential (Giri et al. 2005), enzyme (Radhakrishnan et al. 2015) and light (Tang et al. 2015), etc. For instance, Chang et al. fabricated a $\mathrm{pH}$-sensitive drug delivery system by modifying the surface of silica nanoparticles with polydopamine. The anti-cancer drug desipramine contained in $\mathrm{pH}$-triggered silica nanoparticles can be released in low-pH cellular conditions (Chang et al. 2016). Compared to free desipramine, $\mathrm{pH}$-sensitive silica nanoparticles showed significantly higher toxicity and inhibitory effects to cancer cells. Lipid-coated silica nanoparticles, a lipid bilayer surrounding silica nanoparticles containing anti-cancer drugs, have been investigated recently, and less drug leakage, slower rate of drug release, and more substantial in vivo therapeutic effects where observed (Meng et al. 2015; Liu et al. 2016; Choi et al. 2016) .

\section{Dendrimers}

Dendrimers are 3-dimensional, highly branched monodispersed nanoscale macromolecules (Sharma et al. 2017). Generally, dendrimers consist of an initiator core, branches which emanate from the core and functional end groups on the outermost layer (Gupta et al. 2010). Dendrimers have been considered as a promising nanomaterial for targeted delivery and diagnostic imaging agents, due to their unique properties, such as the monodispersity, internal cavities and modifiable functional end groups (Sharma et al. 2017). The cavity in the hydrophobic core and the multivalent surface allow dendrimers to effectively load hydrophobic and hydrophilic compounds, respectively (Hu et al. 2010). Varied dendrimer-based MRI, X-ray and CT contrast agents have been developed by linking contrast agents to dendrimers with design formulations (Sharma et al. 2017). In addition to diagnostic agents, functionalized and ligand-anchored dendrimers have shown outstanding ability to target drug delivery. The ligands that have been used with dendrimers include folic acid (Hilgenbrink \& Low 2005), biotin (Yang et al. 2009), N-acetyl-glucosamin (Vannucci et al. 2003) and riboflavin, etc. (Sharma et al. 2017) Due to the definition of dendrimers, the formulations of dendrimers are various, such as PAMAM(Buczkowski et al. 2011), polyglycerol (Lee \& Ooya 2012), PPI (Jain et al. 2015), polyester (Morgan et al. 2006) and nucleic acid (Taghdisi et al. 2016), etc. (Sharma et al. 2017) For instance, Taghdisi et al. fabricated an aptamer-base DNA dendrimer containing the anti-cancer drug epirubicin, and targeted drug delivery and considerable in vivo tumour inhibiting effects were observed (Taghdisi et al. 2016).

Several major types of nanovectors, including liposomes, polymeric, metal-based, silicon and silica-based nanoparticles, and dendrimers which have been applied in clinical or preclinical trials for targeted delivery of anti-cancer drugs, have been briefly introduced above. Moreover, oceans of novel nanoparticles are currently under study and the systematically combination of varied nanomaterial will provide a very large number of options for selecting highly-specific and highly-efficient therapeutic nanovectors according to individual patient. On the other hands, to develop more selective and less toxic cancer therapies, nanoparticles have been applied to combine several therapeutic interventions, including photodynamic (Roblero-Bartolon \& Ramon-Gallegos 2015; Agostinis et al. 2011; Chatterjee et al. 2008), sonodynamic (Xu et al. 2016; Rengeng et al. 2017) and radiotherapy (Hainfeld \& Dilmanian 2010), etc., as sensitisers or imaging agent or both. The following sections will review the recent advance in the combination of nanoparticles with existing anti-cancer interventions. 


\section{Nano-Sensitisers}

\section{Photosensitisers}

Photodynamic therapy (PDT), known as photochemotherapy of tumours, is a clinically approved technology used in both the treatment of neoplasm and other diseases (Agostinis et al. 2011; Dougherty et al. 1978). PDT is able to selectively kill cancer cells by the administration of a light-sensitive photosensitizer (PS, non-toxic dye) followed by local irradiation at an appropriate wavelength (Agostinis et al. 2011; Ahn et al. 2014; Oliveira et al. 2011). PSs are usually single compounds that can be triggered at the appropriate wavelength, have no cytotoxicity until being activated and can be rapidly cleared by health tissues, such as Verteporfin (VP) and Rose Bengal (RB), which are well-known PSs used for PDT. After the absorption of light, the PSs are converted from the ground state to singlet state and later to the triplet state. Triplet state PSs can transfer the energy or charge to oxygen molecules to create reactive oxygen species, such as superoxide anion radicals, hydrogen peroxide, oxidant hydroxyl radicals and especially singlet oxygen, which plays a key role in PDT (Kochevar \& Redmond 2000; Foote 1991; DeRosa \& Crutchley 2002).

Nanoparticles have been utilized in PDT as PSs, light transducers and PS carriers etc. (Roblero-Bartolon \& Ramon-Gallegos 2015; Agostinis et al. 2011; Chatterjee et al. 2008). Nanoparticles that can absorb and/or transfer light energy and generate ROS by themselves after irradiation activation include $\mathrm{TiO}_{2}$ nanoparticles (Rengeng et al. 2017), quantum dots (Chatterjee et al. 2008), silicon nanoparticles (Agostinis et al. 2011), silica nanoparticles (Kim et al. 2017; W. H. Chen et al. 2017) and conjugated polymer nanoparticles (Qian et al. 2017), etc. Nanoparticles are also promising carriers for PSs, as they can be designed to provide excellent biocompatibility, improve the stability, increase the efficiency of delivery to targeted tissues, overcome the blood-brain barrier (BBB) (Dixit et al. 2015) or cell-membrane transporters (Roh et al. 2017), and to enhance the generation of singlet oxygen by PSs. (Clement et al. 2017; Kautzka et al. 2017)

For instance, Ting Yin et al. assembled $\mathrm{Fe}_{3} \mathrm{O}_{4} @$ polymer-NPO/PEG-Glc@Ce6 nanoprobes which have prolonged residence time in tumour tissue and reduced accumulation in the normal organs, due to their targeting ability, ultra-small size and high stability (Yin et al. 2017). Polymeric nanoparticles are capable of adsorbing on cell surfaces and accumulate in cells due to cellular uptake mechanisms. Therefore, they are used for delivery and controlled-release of various PSs, which can provide high loading efficiency and improve the stability of PSs, such as bovine serum albumin NPs (Khanadeev et al. 2017), PLA/PLGA NPs (Ricci-Júnior \& Marchetti 2006; Konan et al. 2003; Konan-Kouakou \& Boch 2005), pPFPA NPs (Y. Liu et al. 2015). Other PS carriers include gold, iron oxide, ceramic (silica) and polyacrylamide NPs, and they can be modified with targeting peptides or antibodies and have high efficiency of loading (Chatterjee et al. 2008).

The delivery of therapeutic drugs to brain tumours is limited by the BBB. As a promising delivering vector, gold nanoparticles have been demonstrated to be capable of efficiently crossing the BBB. PS-loaded AuNPs functionalised with epidermal growth factor peptide (Meyers \& Cheng 2015) and transferrin peptide (Dixit et al. 2015) etc. for brain cancer photodynamic therapy have shown significant improvements in 
cancer cell killing and accumulation of Pc 4 in brain tumour tissues, both in vitro and vivo. Roh et al. reported that polymeric nanoparticles can be used to encapsulate Chlorin-based photosensitizers, another drug commonly used in PDT, to overcome the efflux by cell-membrane transporters and increase the intracellular concentration of the PS (Roh et al. 2017). The conjugation of VP and RB to CeF3 and gold nanoparticles has been proven increase the efficiency of singlet oxygen generation by PSs (Clement et al. 2017). Gold nanoparticles were also found to enhance the generation of singlet oxygen while encapsulated in light-triggered liposomes containing RB. The local electric field enhancement caused by the AuNPs could be the potential reason (Kautzka et al. 2017).

Due to the limited penetration of light through human tissues, the efficiency of PDT can only be guaranteed for the superficial layer of the treated tissues. In a recent study, Ozdemir et al. demonstrated that persistent luminescent nanoparticles conjugated with PSs allow a delayed emission and induce the generation of singlet oxygen without any need for light exposure (Ozdemir et al. 2017). Near-infrared light has stronger penetrating power than UV-visible light, which can be used to overcome the limitation of penetration depth in PDT (Zhou et al. 2010; Hirsch et al. 2003). Although photosensitizers such as aluminum sulfophthalocyanine (790 nm) (Kogan et al. 1999) and indocyanine green (absorption at 800 to $810 \mathrm{~nm}$ ) (Omar et al. 2008; Funayama et al. 2012) can absorb near-infrared light directly, the generation of the triplet state by these photosensitizers appears lower compared to other photosensitizers that use UV or visible light for the activation (Omar et al. 2008; Bäumler et al. 1999; Allison et al. 2004). Alternative options are provided by upconverting nanoparticles, which are modified nanosized composite materials such as $\mathrm{NaYF} 4 \mathrm{Yb}$ and $\mathrm{Er} / \mathrm{Tm}$ that can convert long wavelength light (near infrared) to shorter wavelength light that activates the associated PS, (Chatterjee et al. 2008; Dou et al. 2015).

Multifunctional NPs can also facilitate the combination of PDT with other physical treatments, such as chemotherapy (Kautzka et al. 2017; Lee \& Chang 2017), photothermal therapy (Yang et al. 2017; Song et al. 2015), radiation therapy and imaging (Ai et al. 2014; Manuscript 2009; Terentyuk et al. 2014). For example, Chadwick et al. described a hybrid NP consisting of a gold nanorod core and a silica shell containing hematoporphyrin (HP) as PS (Terentyuk et al. 2014). It has been found that the 633-nm laser-induced generation of singlet oxygen and 808-nm laser-induced production of heat can cause combined effects to solid tumours in a rat model and results in dramatic tumoricidal effect compared with PDT alone (Terentyuk et al. 2014). It has been reported that polypyrole nanoparticles, a type of conjugated polymer, linked with photosensitizer Ce6, were able to induce both photodynamic therapy (PDT) and photothermal therapy (PTT) in the targeted tissues and showed considerable synergistic effects compared with PDT or PTT alone (Song et al. 2015). Yang et al. assembled hollow manganese dioxide nanoparticles loaded with both photosensitizer Ce6 and anticancer drugs DOX which could release therapeutic molecules within the low-pH tumour microenvironment and showed a significant synergistic therapeutic effect in vivo (Yang et al. 2017).

\section{Sonosensitisers}

When ultrasonic waves propagate in a medium, the molecules will oscillate about their mean position, resulting in a change of the average distance between them (Lorimer \& 
Mason 1987), leading to alternating cycles of rarefaction and compression (reduced and increased pressure respectively) (Kennedy 2005). During the expansion phase, the average distance between the molecules becomes greater and it becomes easier to form a cavity and draw gas/vapour out of solution to create bubbles (Flynn 1964). Subsequent ultrasonic waves will cause the oscillation of gas bubbles, termed acoustic cavitation, which can be divided into two categories (stable cavitation and inertial cavitation) (Brennen 1995). Using relatively lower-intensity ultrasound, the bubbles are able to oscillate in size repeatedly, which is called stable cavitation. Inertial cavitation is the rapid expansion and then sudden collapse of bubbles upon exposure to higherintensity ultrasound. The violent collapse can cause shock waves, local energy release, temperature rises and generation of reactive oxygen species (ROS) at a microscopic level (Husseini et al. 2005; Kennedy 2005). This can be harnessed to promote cancer cell inactivation (Wood \& Sehgal 2015). Advantages of this technological approach include directionality, relatively high resolution, the ability to focus and target a specific region and the ability to penetrate biological tissue and reach much deeper targeted tumours compared to PDT.

Ultrasound has been utilized in various neoplasm treatment applications, which can be broadly classified into two types: high intensity ultrasound and low intensity ultrasound. It has been suggested that high intensity ultrasound treatmentssuch as ultrasonic surgical instruments and high intensity focused ultrasound (HIFU) (Yu et al. 2004). mainly cause structural alterations in tissue and favours heat-production, In contrast, low intensity ultrasound (LIU) treatments, mostly modulate the functions of tissue and achieve cell killing effect together with other assistants, such as sensitizing or chemotherapeutic molecules via acoustic cavitation (Wood \& Sehgal 2015; Yu et al. 2004) LIU is safe to normal tissues and the implementation of it is simpler and less expensive (Wood \& Sehgal 2015). Therefore, LIU has been applied in several promising and emerging neoplasm treatments, which include ultrasound mediated gene therapy (Newman \& Bettinger 2007; Hernot \& Klibanov 2008), ultrasound mediated chemotherapy (Jr \& Tachibana 2012; Li et al. 2013), antivascular ultrasound therapy (Wood \& Sehgal 2015) and sonodynamic therapy.

Sonodynamic therapy (SDT): ultrasound induced inertial cavitation can produce ROS together with sonosensitizers and leads to a series of molecular reactions and finally cell death (Wood \& Sehgal 2015). The sonosensitizers, which can be excited by inertial cavitation and generate ROS and other free radicals, are similar to photosensitizers and have been developed extensively in recent years (Chen et al. 2014).

Due to the specific advantages of nanoparticles, a variety of nanoparticles have been introduced into the development of SDT, as high-performance sonosensitizers or efficient carriers for the sonosensitizer. There have been some promising nano materials developed as sonosensitizers, such as gold, $\mathrm{TiO}_{2}, \mathrm{Fe}_{3} \mathrm{O}_{4}$, Carbon fluoroxide, porous silicon, silver and silver copper nanoparticles, etc. (Xu et al. 2016; Rengeng et al. 2017) For instance, $\mathrm{TiO}_{2}$ nanoparticles, as an emerging nano material, also known as a photosensitizer, have high biocompatibility, great stability, small molecular weight and accessibility to cytomembrane. After being treated with ultrasound, the $\mathrm{TiO}_{2} \mathrm{NPs}$ inside cells can generate active hydroxyl ions which can kill target cells in vitro and in animal models (Rengeng et al. 2017). Chen et al. have recently reported mitochondria targeting liposomes for sonosensitizer delivery . In this study, the liposomes were applied as 
nanocarriers for the hematoporphyrin monomethyl ether (HMME), a typical hydrophobic sonosensitizer as well as photosensitizer. After the accumulation of the liposomes in the mitochondria, the lipid in liposomes can be oxidised by ultrasound, causing the release of HMME. HMME can then be actived by the ultrasound and produce ROS inside the mitochondria, which can kill cancer cells more effectively (M. Chen et al. 2017). Miyoshi and colleagues reported the combination of sonodynamic therapy (SDT) and photodynamic therapy (PDT) (Miyoshi et al. 2016). Using 0.2\% $\mathrm{TiO}_{2}$ nanoparticles as sonosensitizers and 5-aminolevulinic acid as photosensitizers in a mouse model, the synergy of SDT and PDT was demonstrated to result in significantly greater antitumour activity compared with SDT or PDT alone (Miyoshi et al. 2016).

\section{Radiosensitisers}

Radiation therapy (RT) using high-energy ionizing radiation (IR)is one of the primary treatments for neoplasm. RT is effective in controlling or killing various cancer cells and has been prescribed for a large proportion of cancer patients, as sole treatment or combined with other interventions, for more than 100 years. The mechanisms of action of RT include the generation of free radicals or the direct deposition of energy by IR. Due to photoelectric effects and Compton effects, the track of IR induces ionization and excitation in tissue (Rehman et al. 2015). In cells, the electrons interact with water and generate free radicals, including reactive oxygen and nitrogen species (ROS and RNS) (Mikkelsen \& Wardman 2003). Damage is induced by the ROS/RNS, causing oxidative stress and lesions of cellular macromolecules, including DNA, protein and lipids (Nikitaki et al. 2016). Meanwhile, the cell killing effect is also related to the direct deposition of energy by IR, which is highly penetrating and able to cause irreparable damage to genetic material even at low dose (Rothkamm \& Löbrich 2003). However, due to the high cytotoxicity and non-targeted effects of radiation, the radiation must be accurately delivered to tumour tissue while sparing normal tissue to improve PFS, OS and ultimately the life quality of patients (Nikitaki et al. 2016).

Currently, RT is highly developed and refined, primarily based on three aspects: dose/fractionation, delivery systems and guiding/monitoring to minimise the side effects and improve treatments. RT has also been demonstrated to have strong synergic or additive effects with a great variety of neoplasm treatments, such as chemotherapy (Bentzen et al. 2007; Plastaras et al. 2007; Régnard \& Bräuer-Krisch 2008; Lund et al. 2000), nanotechnology (Hainfeld \& Dilmanian 2010), gene therapy (Badie et al. 1999; Cook et al. 2004; Gupta et al. 2003), hyperthermia (Kampinga 2006), photodynamic therapy and immunotherapy (Simone et al. 2015). Nanoparticles have been used as imaging agents for cancer diagnostics, which provides excellent guiding/monitoring for RT. Nano-sensitizers also show considerable synergistic effects combined with radiotherapies. Various nano materials have been evaluated in combination with RT in cancer therapy, such as gold (Tailor et al. 2015; Wu et al. 2016), gadolinium (Wozny et al. 2017), hafnium, bismuth (Detappe et al. 2016), copper (Liu et al. 2017) and magnetic iron oxide nanoparticles (Attaluri et al. 2015). Metal-base nanoparticles have great potential as radiosensitizers, mainly due to the increased radiation interaction cross-section of cancer cells, causing the emission of abundant Auger electrons and short-range photoelectrons and resulting in hyperthermia and damage to the cancer cells (Kunjachan et al. 2015; Hainfeld et al. 2014). The biological mechanisms involved in nanoparticle radiosensitization include oxidative stress and DNA damage induction, 
cell cycle interruption and bystander effects (Rosa et al. 2017). For instance, using gadolinium chelated on silica nanoparticles, Detappe et al. developed an advanced multimodal gadolinium chelated silica nanoparticles ( $\mathrm{SiGdNP}$ ), as both imaging agents and radiosensitizers, and evaluated the synergic effects in cynomolgus monkeys and mice models injected with human pancreatic adenocarcinoma (capan-1) cells. After being injected with SiGdNPs, the subjects were treated with radiation and observed for various parameters then euthanasised after two weeks. It was shown that the cancer cell damage (DNA damage), tumour ablation, suppression of growth and overall survival were significantly improved by the combination of SiGdNPs and radiation (Detappe et al. 2016).

\section{Conclusion}

Extensive efforts have been made in the war against cancer in the past decades, however, the treatment of cancer is challenging due to the tumor heterogeneity and varied patient characteristics, and widespread application of advanced stage cancer treatment and early diagnosis of cancer are still the highest aspirations. In the development of successful interventions to cure cancer, nanomaterials have played key roles. In last two decades, a variety of nanomaterials have been greatly expanded as well as highly diversified and multi-functionalized. Some of these nanomaterials were determined as lead candidates to delivery chemotherapeutics to tumors and have passed preclinical trials and successfully applied to clinical. EPR-based tumor targeting and then, especially, active targeting was introduced, which highly increased the efficiency and specificity of drug delivery into tumors.

Moreover, there is great promise in the combination of nanomaterial with other therapeutic interventions, such as photodynamic, sonodynamic and radiotherapy, etc. Due to the excellent loading capacity of nanocarriers and multifunctionality of their ligand or themselves, nanoparticles can be applied in cancer treatments, as both carriers, sensitisers and imaging agents, which are capable of providing advance diagnosis as well as diversified treatments. Therefore, nanomedicine has been considered as an important and promising intervention in personalized medicine, including but not limited to cancer therapy nowadays.

Despite the promise of nanomedicine, there are several challenges to overcome for more successful clinical translation. In-depth understanding of the basic mechanism that underlies the anti-cancer effects of nanomedicine is required. The community should also make efforts on low-cost commercial manufacturing of homogenous biomedical nanomaterials. Then, nanomaterials will benefit the whole mankind in the near further.

\section{References}

Agostinis, P. et al., 2011. Photodynamic Therapy of Cancer : An Update. American Cancer Society, 61, pp. 250-281.

Ahn, J.C. et al., 2014. Cellular uptake of 9-hydroxypheophorbide-?? and its photoactivation to induce ER stress-related apoptosis in human cervical cancer cells. Lasers in Medical Science, 29(1), pp.289-299. Available at: http://link.springer.com/article/10.1007/s10103-013-1331-4 [Accessed June 5, 2016]. 
Ai, J. et al., 2014. Multifunctional AS1411-functionalized fluorescent gold nanoparticles for targeted cancer cell imaging and efficient photodynamic therapy. Talanta. Available at: http://www.sciencedirect.com/science/article/pii/S0039914013008011 [Accessed June 5, 2016].

Akhtar, M.J. et al., 2013. Dose-dependent genotoxicity of copper oxide nanoparticles stimulated by reactive oxygen species in human lung epithelial cells. Toxicology and industrial health, 32(5), pp.809-821. Available at: http://journals.sagepub.com/doi/10.1177/0748233713511512 [Accessed November 6, 2017].

Alexis, F. et al., 2008. New frontiers in nanotechnology for cancer treatment. Urologic Oncology: Seminars and Original Investigations, 26(1), pp.74-85.

Alkilany, A.M. \& Murphy, C.J., 2010. Toxicity and cellular uptake of gold nanoparticles: What we have learned so far? Journal of Nanoparticle Research, 12(7), pp.2313-2333.

Allison, R.R. et al., 2004. Photosensitizers in clinical PDT. Photodiagnosis and Photodynamic Therapy, 1(1), pp.27-42. Available at: http://www.sciencedirect.com/science/article/pii/S1572100004000079 [Accessed November 20, 2017].

Anderson, V.C. \& Thompson, D.H., 1992. Triggered release of hydrophilic agents from plasmalogen liposomes using visible light or acid. Biochimica et biophysica acta, 1109, pp.33-42.

Arouri, A. et al., 2013. Lipases, liposomes and lipid-prodrugs. Current Opinion in Colloid and Interface Science, 18(5), pp.419-431.

Attaluri, A. et al., 2015. Magnetic nanoparticle hyperthermia enhances radiation therapy: A study in mouse models of human prostate cancer. International Journal of Hyperthermia, 31(4), pp.359374. Available at: http://www.tandfonline.com/doi/full/10.3109/02656736.2015.1005178.

Badie, B. et al., 1999. Combined radiation and p53 gene therapy of malignant glioma cells. Cancer gene therapy, 6(2), pp.155-62. Available at: http://www.ncbi.nlm.nih.gov/pubmed/16644107 [Accessed May 7, 2016].

Bae Ki, H., Mok, H. \& Park Tae, G., 2008. Synthesis, characterization, and intracellular delivery of reducible heparin nanogels for apoptotic cell death. Biomaterials (Guildford, Engl.), 29(23), pp.3376-3383. Available

at: http://www.sciencedirect.com/science/article/pii/S0142961208002834 [Accessed May 7, 2016].

Baetke, S.C., Lammers, T. \& Kiessling, F., 2015. Applications of nanoparticles for diagnosis and therapy of cancer. British Journal of Radiology, 88(1054). Available at: http://www.birpublications.org/doi/abs/10.1259/bjr.20150207 [Accessed May 7, 2016].

Bakhshizadeh, M. et al., 2017. TiO2-based mitoxantrone imprinted poly (methacrylic acid-copolycaprolctone diacrylate) nanoparticles as a drug delivery system. Current pharmaceutical design, 23(999), pp.1-1. Available at: http://www.eurekaselect.com/150099/article [Accessed November 6, 2017].

Bäumler, W. et al., 1999. Photo-oxidative killing of human colonic cancer cells using indocyanine green and infrared light. British Journal of Cancer, 80(3-4), pp.360-363. Available at: http://www.nature.com/articles/6690363 [Accessed November 20, 2017].

Bennet, D. \& Kim, S., 2014. Polymer Nanoparticles for Smart Drug Delivery. In Application of Nanotechnology in Drug Delivery. InTech. Available at: http://www.intechopen.com/books/application-of-nanotechnology-in-drug-delivery/polymernanoparticles-for-smart-drug-delivery [Accessed November 6, 2017].

Bentzen, S., Harari, P. \& Bernier, J., 2007. Exploitable mechanisms for combining drugs with radiation: concepts, achievements and future directions. Nature clinical practice Oncology. Available at: http://www.nature.com/nrclinonc/journal/v4/n3/full/ncponc0744.html [Accessed June 6, 2016].

Bibi, S. et al., 2012. Trigger release liposome systems: local and remote controlled delivery? Journal of Microencapsulation, 29(3), pp.262-276. Available at: http://www.tandfonline.com/doi/full/10.3109/02652048.2011.646330.

Boussif, O. et al., 1995. A versatile vector for gene and oligonucleotide transfer into cells in culture and in vivo: polyethylenimine. Proceedings of the National Academy of Sciences of the United States of America, 92(16), pp.7297-301. Available at: http://www.ncbi.nlm.nih.gov/pubmed/7638184\%5Cnhttp://www.pubmedcentral.nih.gov/articlere nder.fcgi?artid=PMC41326 [Accessed May 7, 2016].

Bregoli, L. et al., 2016. Nanomedicine applied to translational oncology: A future perspective on cancer treatment. Nanomedicine: Nanotechnology, Biology, and Medicine, 12(1), pp.81-103. Available 
at: http://dx.doi.org/10.1016/j.nano.2015.08.006.

Brennen, C., 1995. Cavitation and bubble dynamics, 1995. Oxford University Press. Available at: https://scholar.google.com/scholar?hl=en\&q=C.E.+Brennen\%2C+Cavitation+and+Bubble+Dyna mics+\%280xford+University\&btnG=\&as_sdt=1\%2C5\&as_sdtp=\#0 [Accessed June 6, 2016].

Buczkowski, A. et al., 2011. Interaction between PAMAM-NH 2 G4 dendrimer and 5-fluorouracil in aqueous solution. International Journal of Pharmaceutics, 408(1-2), pp.266-270. Available at: http://www.sciencedirect.com/science/article/pii/S037851731100130X [Accessed November 6, 2017].

Casasus, R. et al., 2004. Toward the development of ionically controlled nanoscopic molecular gates. Journal of the American Chemical Society, 126(28), pp.8612-8613. Available at: http://pubs.acs.org/doi/abs/10.1021/ja048095i [Accessed November 6, 2017].

Chan, M. \& Almutairi, A., 2016. Nanogels as imaging agents for modalities spanning the electromagnetic spectrum. Mater. Horiz., 3(1), pp.21-40. Available at: http://www.scopus.com/inward/record.url?eid=2-s2.0-84952046046\&partnerID=tZOtx3y1.

Chang, D. et al., 2016. Polydopamine-based surface modification of mesoporous silica nanoparticles as $\mathrm{pH}$-sensitive drug delivery vehicles for cancer therapy. Journal of Colloid and Interface Science, 463, pp.279-287. Available

at: http://www.sciencedirect.com/science/article/pii/S0021979715303118 [Accessed November 6, 2017].

Chatterjee, D.D.K., Fong, L.L.S. \& Zhang, Y., 2008. Nanoparticles in photodynamic therapy: An emerging paradigm. Advanced Drug Delivery Reviews, 60(15), pp.1627-1637. Available at: http://dx.doi.org/10.1016/j.addr.2008.08.003 [Accessed June 5, 2016].

Chen, H. et al., 2014. Recent progress in development of new sonosensitizers for sonodynamic cancer therapy. Drug Discovery Today, 19(4), pp.502-509. Available at: http://dx.doi.org/10.1016/j.drudis.2014.01.010.

Chen, H. et al., 2017. Rethinking cancer nanotheranostics. Nature Reviews Materials, 2(7), p.17024. Available at: http://www.nature.com/articles/natrevmats201724.

Chen, M. et al., 2017. Ultrasound triggered drug delivery for mitochondria targeted sonodynamic therapy. Journal of Drug Delivery Science and Technology, 39, pp.501-507. Available at: http://linkinghub.elsevier.com/retrieve/pii/S1773224717301776.

Chen, W.H. et al., 2017. Mesoporous silica-based versatile theranostic nanoplatform constructed by layer-by-layer assembly for excellent photodynamic/chemo therapy. Biomaterials, 117, pp.54-65. Available at: http://www.sciencedirect.com/science/article/pii/S0142961216306883 [Accessed November 6, 2017].

Cheng, X. et al., 2014. Synergistic effect of gold nanoparticles and cold plasma on glioblastoma cancer therapy. Journal of Physics D: Applied Physics, 47(33), p.335402. Available at: http://stacks.iop.org/0022-

3727/47/i=33/a=335402?key=crossref.9b444f36ef2dd3a227a5602d555eb1b1.

Choi, J.Y. et al., 2016. PEGylated lipid bilayer-supported mesoporous silica nanoparticle composite for synergistic co-delivery of axitinib and celastrol in multi-targeted cancer therapy. Acta Biomaterialia, 39, pp.94-105.

Clement, S. et al., 2017. Nanoparticle-mediated singlet oxygen generation from photosensitizers. Journal of Photochemistry and Photobiology A: Chemistry, 332, pp.66-71. Available at: http://dx.doi.org/10.1016/j.jphotochem.2016.08.009.

Connor, E.E. et al., 2005. Gold nanoparticles are taken up by human cells but do not cause acute cytotoxicity. Small, 1(3), pp.325-327. Available at: http://dx.doi.org/10.1002/smll.200400093 [Accessed May 7, 2016].

Cook, T. et al., 2004. Nitric oxide and ionizing radiation synergistically promote apoptosis and growth inhibition of cancer by activating p53. Cancer Research. Available at: http://cancerres.aacrjournals.org/content/64/21/8015.short [Accessed June 6, 2016].

Couvreur, P. \& Vauthier, C., 2006. Nanotechnology: Intelligent design to treat complex disease,

Date, A. a. et al., 2012. Translating Nanotechnology from Bench to Pharmaceutical Market: Barriers, Success, and Promises. Journal of Drug Delivery, 2012, pp.1-2. Available at: http://www.ncbi.nlm.nih.gov/pmc/articles/PMC3382950/ [Accessed May 7, 2016].

DeRosa, M.C. \& Crutchley, R.J., 2002. Photosensitized singlet oxygen and its applications. Coordination 
Chemistry Reviews, 233-234, pp.351-371. Available at: http://www.sciencedirect.com/science/article/pii/S0010854502000346 [Accessed November 6, 2017].

Deshpande, P.P., Biswas, S. \& Torchilin, V.P., 2013. Current trends in the use of liposomes for tumor targeting. Nanomedicine (London, England), 8(9), pp.1509-28. Available at: http://www.ncbi.nlm.nih.gov/pubmed/23914966\%5Cnhttp://www.pubmedcentral.nih.gov/articler ender.fcgi?artid=PMC3842602.

Detappe, A. et al., 2016. Advanced multimodal nanoparticles delay tumor progression with clinical radiation therapy. Journal of Controlled Release, 238(July), pp.103-113. Available at: http://dx.doi.org/10.1016/j.jconrel.2016.07.021.

Dixit, S. et al., 2015. Transferrin receptor-targeted theranostic gold nanoparticles for photosensitizer delivery in brain tumors. Nanoscale, 7(5), pp.1782-1790. Available at: http://xlink.rsc.org/?DOI=C4NR04853A.

Dou, Q.Q. et al., 2015. Effective near-infrared photodynamic therapy assisted by upconversion nanoparticles conjugated with photosensitizers. International Journal of Nanomedicine, 10, pp.419-432.

Dougherty, T.J. et al., 1978. Photoradiation Therapy for the Treatment of Malignant Tumors. Cancer Research, 38(8), pp.2628-2635. Available at: http://www.nature.com/doifinder/10.1038/nrc1071 [Accessed November 6, 2017].

Duchene, D., Cavalli, R. \& Gref, R., 2016. Cyclodextrin-based Polymeric Nanoparticles as Efficient Carriers for Anticancer Drugs. Current Pharmaceutical Biotechnology, 17(3), pp.248-255. Available

at: http://www.ingentaconnect.com/content/ben/cpb/2016/00000017/00000003/art00006 [Accessed November 6, 2017].

Eckmann, D.M. et al., 2014. Nanogel Carrier Design for Targeted Drug Delivery. J. Mater. Chem. B, 2(46), pp.8085-8097. Available at: http://pubs.rsc.org/en/content/articlehtml/2014/tb/c4tb01141d\%5Cnhttp://www.ncbi.nlm.nih.gov/ pubmed/25485112.

El-Sayed, I.H., Huang, X. \& El-Sayed, M.A., 2006. Selective laser photo-thermal therapy of epithelial carcinoma using anti-EGFR antibody conjugated gold nanoparticles. Cancer Letters, 239(1), pp.129-135. Available at: http://www.sciencedirect.com/science/article/pii/S0304383505007378 [Accessed May 7, 2016].

Elhissi, A.M. et al., 2012. Carbon nanotubes in cancer therapy and drug delivery. J Drug Deliv, 2012, p.837327. Available at: http://www.ncbi.nlm.nih.gov/pubmed/22028974 [Accessed May 7, 2016].

Fairhurst, D. \& Lee, R., 2012. Observations on nanotechnologybased drug delivery approaches: translating nanotechnology from bench to pharmaceutical market: barriers, success, and. The American Pharmaceutical Review. Available at: http://www.particlesciences.com/docs/observations_on_nanotechnology_based_drug_delivery.pd f [Accessed May 7, 2016].

Feng, Y. et al., 2016. The application of mesoporous silica nanoparticle family in cancer theranostics. Coordination Chemistry Reviews, 319, pp.86-109. Available at: http://dx.doi.org/10.1016/j.ccr.2016.04.019.

Ferrari, M., 2005. Cancer nanotechnology: opportunities and challenges. Nature Reviews Cancer, 5(3), pp.161-171. Available at: http://www.nature.com/doifinder/10.1038/nrc1566.

Ferrari, M. \& Phelps, M., 2005. Nanotechnology Takes Aim at Cancer. Science, 310, p.1132. Available at: http://cml.harvard.edu/assets/Science_18Nov05.pdf [Accessed May 7, 2016].

Flynn, H., 1964. Physics of acoustic cavitation in liquids. Physical acoustics. Available at: https://scholar.google.com/scholar?q=Physics+of+acoustic+cavitation+in+liquids\&btnG=\&hl=en \&as_sdt=0\%2C5\#0 [Accessed June 5, 2016].

Foldbjerg, R. et al., 2009. PVP-coated silver nanoparticles and silver ions induce reactive oxygen species, apoptosis and necrosis in THP-1 monocytes. Toxicology Letters, 190(2), pp.156-162. Available at: http://www.sciencedirect.com/science/article/pii/S0378427409012211 [Accessed November 6, 2017].

Foote, C.S., 1991. DEFINITION OF TYPE I and TYPE II PHOTOSENSITIZED OXIDATION. Photochemistry and Photobiology, 54(5), pp.659-659. Available at: http://doi.wiley.com/10.1111/j.1751-1097.1991.tb02071.x [Accessed November 3, 2017]. 
Funayama, T. et al., 2012. Photodynamic Therapy with Indocyanine Green Injection and Near-Infrared Light Irradiation Has Phototoxic Effects and Delays Paralysis in Spinal Metastasis. Photomedicine and Laser Surgery, 30(1), pp.47-53. Available at: http://www.liebertonline.com/doi/abs/10.1089/pho.2011.3080 [Accessed November 20, 2017].

Giri, S. et al., 2005. Stimuli-responsive controlled-release delivery system based on mesoporous silica nanorods capped with magnetic nanoparticles. Angewandte Chemie - International Edition, 44(32), pp.5038-5044. Available at: http://doi.wiley.com/10.1002/anie.200501819 [Accessed November $6,2017]$.

Godin, B. et al., 2011. Multistage nanovectors: From concept to novel imaging contrast agents and therapeutics. Accounts of Chemical Research, 44(10), pp.979-989. Available at: http://pubs.acs.org/doi/abs/10.1021/ar200077p [Accessed November 6, 2017].

Goodman, C.M. et al., 2004. Toxicity of gold nanoparticles functionalized with cationic and anionic side chains. Bioconjugate Chemistry, 15(4), pp.897-900.

Gunawan, R.C. \& Auguste, D.T., 2010. The role of antibody synergy and membrane fluidity in the vascular targeting of immunoliposomes. Biomaterials, 31(5), pp.900-907.

Gupta, A., Cerniglia, G. \& Mick, R., 2003. Radiation sensitization of human cancer cells in vivo by inhibiting the activity of PI3K using LY294002. International Journal of .... Available at: http://www.sciencedirect.com/science/article/pii/S0360301603002141 [Accessed June 6, 2016].

Gupta, U. et al., 2010. Ligand anchored dendrimers based nanoconstructs for effective targeting to cancer cells. International Journal of Pharmaceutics, 393(1-2), pp.186-197. Available at: http://www.sciencedirect.com/science/article/pii/S0378517310002747 [Accessed November 6, 2017].

Hainfeld, J. \& Dilmanian, F., 2010. Gold nanoparticles enhance the radiation therapy of a murine squamous cell carcinoma. Physics in medicine ... Available at: http://iopscience.iop.org/article/10.1088/0031-9155/55/11/004/meta [Accessed June 6, 2016].

Hainfeld, J.F. et al., 2014. Gold nanoparticle hyperthermia reduces radiotherapy dose. Nanomedicine: Nanotechnology, Biology, and Medicine, 10(8), pp.1609-1617.

Hernot, S. \& Klibanov, A., 2008. Microbubbles in ultrasound-triggered drug and gene delivery. Advanced drug delivery reviews. Available at: http://www.sciencedirect.com/science/article/pii/S0169409X08000811 [Accessed June 6, 2016].

Hilgenbrink, A.R. \& Low, P.S., 2005. Folate receptor-mediated drug targeting: From therapeutics to diagnostics. Journal of Pharmaceutical Sciences, 94(10), pp.2135-2146. Available at: http://linkinghub.elsevier.com/retrieve/pii/S0022354916318688 [Accessed November 6, 2017].

Hirsch, L.R. et al., 2003. Nanoshell-mediated near-infrared thermal therapy of tumors under magnetic resonance guidance. Proceedings of the National Academy of Sciences, 100(23), pp.13549-13554. Available at: http://www.ncbi.nlm.nih.gov/pubmed/14597719 [Accessed November 20, 2017].

Hu, C.-M.J., Aryal, S. \& Zhang, L., 2010. Nanoparticle-assisted combination therapies for effective cancer treatment. Therapeutic Delivery, 1(2), pp.323-334.

Husseini, G.A. et al., 2005. The role of cavitation in acoustically activated drug delivery. Journal of Controlled Release, 107(2), pp.253-261.

Jack Hu, C.-M. \& Zhang, L., 2009. Therapeutic Nanoparticles to Combat Cancer Drug Resistance. Current Drug Metabolism, 10, pp.836-841.

Jain, N.K. et al., 2015. The development, characterization and in vivo anti-ovarian cancer activity of poly(propylene imine) (PPI)-antibody conjugates containing encapsulated paclitaxel. Nanomedicine: Nanotechnology, Biology, and Medicine, 11(1), pp.207-218. Available at: http://www.sciencedirect.com/science/article/pii/S1549963414005292 [Accessed November 6, 2017].

Jain, P.K., ElSayed, I.H. \& El-Sayed, M.A., 2007. Au nanoparticles target cancer. Nano Today, 2(1), pp.18-29. Available at: http://www.sciencedirect.com/science/article/pii/S1748013207700166 [Accessed May 7, 2016].

Jr, L.F. \& Tachibana, K., 2012. Use of ultrasound in drug delivery systems: emphasis on experimental methodology and mechanisms. International Journal of Hyperthermia. Available at: http://www.tandfonline.com/doi/abs/10.3109/02656736.2012.668640 [Accessed June 6, 2016].

Kaasalainen, M. et al., 2015. Electrostatic interaction on loading of therapeutic peptide GLP-1 into porous silicon nanoparticles. Langmuir, 31(5), pp.1722-1729. Available at: 
http://pubs.acs.org/doi/abs/10.1021/la5047047 [Accessed November 6, 2017].

Kampinga, H., 2006. Cell biological effects of hyperthermia alone or combined with radiation or drugs: a short introduction to newcomers in the field. International journal of hyperthermia. Available at: http://www.tandfonline.com/doi/abs/10.1080/02656730500532028 [Accessed June 6, 2016].

Karanth, H. \& Murthy, R.S.R., 2007. pH-Sensitive liposomes-principle and application in cancer therapy. Journal of Pharmacy and Pharmacology, 59(4), pp.469-483. Available at: http://doi.wiley.com/10.1211/jpp.59.4.0001.

Katiyar, S.S. et al., 2015. Co-delivery of rapamycin- and piperine-loaded polymeric nanoparticles for breast cancer treatment. Drug Delivery, pp.1-9. Available at: http://www.tandfonline.com/doi/full/10.3109/10717544.2015.1039667 [Accessed November 6, 2017].

Kautzka, Z. et al., 2017. Light-triggered liposomal cargo delivery platform incorporating photosensitizers and gold nanoparticles for enhanced singlet oxygen generation and increased cytotoxicity. International Journal of Nanomedicine, 12, pp.969-977.

Kennedy, J.E., 2005. High-intensity focused ultrasound in the treatment of solid tumours. Nature Reviews Cancer, 5(April), pp.321-327.

Khanadeev, V. et al., 2017. Bovine serum albumin nanoparticles loaded with Photosens photosensitizer for effective photodynamic therapy V. V. Tuchin et al., eds., 10336(8452), pp.1-7. Available at: http://proceedings.spiedigitallibrary.org/proceeding.aspx?doi=10.1117/12.2269159 [Accessed November 3, 2017].

Kim, J. et al., 2017. Continuous O 2 -Evolving MnFe 2 O 4 Nanoparticle-Anchored Mesoporous Silica Nanoparticles for Efficient Photodynamic Therapy in Hypoxic Cancer. Journal of the American Chemical Society, 139(32), pp.10992-10995. Available at: http://pubs.acs.org/doi/10.1021/jacs.7b05559 [Accessed November 6, 2017].

Kinsella, J.M. et al., 2011. Enhanced magnetic resonance contrast of $\mathrm{Fe} 3 \mathrm{O} 4$ nanoparticles trapped in a porous silicon nanoparticle host. Advanced Materials, 23(36). Available at: http://onlinelibrary.wiley.com/doi/10.1002/adma.201101877/full [Accessed November 6, 2017].

Kochevar, I.E. \& Redmond, R.W., 2000. Photosensitized production of single oxygen. Methods in Enzymology, 319, pp.20-28. Available at: http://www.sciencedirect.com/science/article/pii/S0076687900190044 [Accessed November 6, 2017].

Kogan, B.Y. et al., 1999. Aluminum sulphophthalocyanine as NIR photosensitizer for PDT. In B. Ehrenberg \& K. Berg, eds. Proceedings of SPIE - The International Society for Optical Engineering. International Society for Optics and Photonics, pp. 148-151. Available at: http://proceedings.spiedigitallibrary.org/proceeding.. aspx?articleid=972242 [Accessed November 20, 2017].

Konan-Kouakou, Y. \& Boch, R., 2005. In vitro and in vivo activities of verteporfin-loaded nanoparticles. Journal of controlled ... Available at: http://www.sciencedirect.com/science/article/pii/S0168365904005887 [Accessed June 5, 2016].

Konan, Y. et al., 2003. Enhanced photodynamic activity of meso-tetra (4-hydroxyphenyl) porphyrin by incorporation into sub-200 nm nanoparticles. European journal of .... Available at: http://www.sciencedirect.com/science/article/pii/S0928098703000174 [Accessed June 5, 2016].

Kong, M.G., Keidar, M. \& Ostrikov, K., 2011. Plasmas meet nanoparticles-where synergies can advance the frontier of medicine. Journal of Physics D: Applied Physics, 44(17), p.174018. Available at: http://stacks.iop.org/00223727/44/i=17/a=174018?key=crossref.c575287f0d5e2832258f2cf71e8fe722.

Kong, W.H. et al., 2012. Cationic lipid-coated gold nanoparticles as efficient and non-cytotoxic intracellular siRNA delivery vehicles. Pharmaceutical Research, 29(2), pp.362-374. Available at: http://link.springer.com/10.1007/s11095-011-0554-y [Accessed November 6, 2017].

Kroto, H.W. et al., 1985. C60: Buckminsterfullerene. Nature, 318(6042), pp.162-163. Available at: http://dx.doi.org/10.1038/318162a0 [Accessed May 7, 2016].

Kumar, A. et al., 2012. Gold nanoparticles functionalized with therapeutic and targeted peptides for cancer treatment. Biomaterials, 33(4), pp.1180-1189.

Kunisawa, J. et al., 2005. Fusogenic liposome delivers encapsulated nanoparticles for cytosolic controlled gene release. Journal of Controlled Release, 105(3), pp.344-353. Available at: http://www.sciencedirect.com/science/article/pii/S0168365905001112 [Accessed November 6, 
2017].

Kunjachan, S. et al., 2015. Nanoparticle Mediated Tumor Vascular Disruption: A Novel Strategy in Radiation Therapy. Nano Letters, 15(11), pp.7488-7496.

de la Rica, R., Aili, D. \& Stevens, M.M., 2012. Enzyme-responsive nanoparticles for drug release and diagnostics. Advanced Drug Delivery Reviews, 64(11), pp.967-978.

Lee, H. \& Ooya, T., 2012. 19F-NMR, 1H-NMR, and fluorescence studies of interaction between 5fluorouracil and polyglycerol dendrimers. Journal of Physical Chemistry B, 116(40), pp.1226312267. Available at: http://pubs.acs.org/doi/abs/10.1021/jp307710b [Accessed November 6, 2017].

Lee, Y.-H. \& Chang, D.-S., 2017. Fabrication, characterization, and biological evaluation of anti-HER2 indocyanine green-doxorubicin-encapsulated PEG-b-PLGA copolymeric nanoparticles for targeted photochemotherapy of breast cancer cells. Scientific Reports, 7, p.46688. Available at: http://www.nature.com/articles/srep46688 [Accessed November 6, 2017].

Li, H. et al., 2013. Potentiation of scutellarin on human tongue carcinoma xenograft by low-intensity ultrasound. PloS one. Available at: http://journals.plos.org/plosone/article?id=10.1371/journal.pone.0059473 [Accessed June 6, 2016].

Li, Z. \& Loh, X.J., 2017. Recent advances of using polyhydroxyalkanoate-based nanovehicles as therapeutic delivery carriers. Wiley Interdisciplinary Reviews: Nanomedicine and Nanobiotechnology, 9(3), pp.19-22. Available at: http://doi.wiley.com/10.1002/wnan.1429 [Accessed November 6, 2017].

Liang, K. et al., 2016. Targeted intracellular protein delivery based on hyaluronic acid-green tea catechin nanogels. Acta Biomaterialia, 33, pp.142-152. Available at: http://linkinghub.elsevier.com/retrieve/pii/S1742706116300113.

Libutti, S.K. et al., 2010. Phase I and pharmacokinetic studies of CYT-6091, a novel PEGylated colloidal gold-rhTNF nanomedicine. Clinical Cancer Research, 16(24), pp.6139-6149. Available at: https://clincancerres.aacrjournals.org/content/16/24/6139.full [Accessed May 7, 2016].

Liu, D. et al., 2013. Co-delivery of a hydrophobic small molecule and a hydrophilic peptide by porous silicon nanoparticles. Journal of Controlled Release, 170(2), pp.268-278. Available at: http://www.sciencedirect.com/science/article/pii/S0168365913003283 [Accessed November 6, 2017].

Liu, D. et al., 2015. Microfluidic assisted one-step fabrication of porous silicon@acetalated dextran nanocomposites for precisely controlled combination chemotherapy. Biomaterials, 39, pp.249-259. Available at: http://www.sciencedirect.com/science/article/pii/S0142961214011569 [Accessed November 6, 2017].

Liu, X. et al., 2016. Irinotecan Delivery by Lipid-Coated Mesoporous Silica Nanoparticles Shows Improved Efficacy and Safety over Liposomes for Pancreatic Cancer. ACS Nano, 10(2), pp.27022715.

Liu, Y. et al., 2015. Modular Synthetic Platform for the Construction of Functional Single-Chain Polymeric Nanoparticles: From Aqueous Catalysis to Photosensitization. Journal of the American Chemical Society, 137(40), pp.13096-13106.

Liu, Z. et al., 2017. Investigation of Copper Cysteamine Nanoparticles as a New Type of Radiosensitiers for Colorectal Carcinoma Treatment. Scientific Reports, 7(1), p.9290. Available at: http://www.nature.com/articles/s41598-017-09375-y.

Lonez, C., Vandenbranden, M. \& Ruysschaert, J.M., 2008. Cationic liposomal lipids: From gene carriers to cell signaling. Progress in Lipid Research, 47(5), pp.340-347. Available at: http://linkinghub.elsevier.com/retrieve/pii/S0163782708000271 [Accessed November 6, 2017].

Lorimer, J. \& Mason, T., 1987. Sonochemistry. Part 1-the physical aspects. Chemical Society Reviews. Available at: http://pubs.rsc.org/en/content/articlepdf/1987/cs/cs9871600239 [Accessed June 5, 2016].

Lund, E., Bastholm, L. \& Kristjansen, P., 2000. Therapeutic synergy of TNP-470 and ionizing radiation: effects on tumor growth, vessel morphology, and angiogenesis in human glioblastoma multiforme xenografts. Clinical Cancer Research. Available at: http://clincancerres.aacrjournals.org/content/6/3/971.short [Accessed June 6, 2016].

Lux, J. et al., 2013. Metal Chelating Crosslinkers Form Nanogels with High Chelation Stability. Journal of Materials Chemistry B, 1(46), pp.6359-6364. Available at: http://www.pubmedcentral.nih.gov/articlerender.fcgi?artid=3910426\&tool=pmcentrez\&renderty 
pe $=$ abstract $[$ Accessed May 7, 2016].

Malaekeh-Nikouei, B. et al., 2008. The enhancement of immunosuppressive effects of cyclosporine A on human T-cells using fusogenic liposomes. Colloids and Surfaces B: Biointerfaces, 67(2), pp.238-244.

Manchun, S. et al., 2015. Enhanced anti-tumor effect of pH-responsive dextrin nanogels delivering doxorubicin on colorectal cancer. Carbohydrate Polymers, 126, pp.222-230. Available at: http://dx.doi.org/10.1016/j.carbpol.2015.03.018 [Accessed May 7, 2016].

Manchun, S., Dass, C. \& Sriamornsak, P., 2012. Targeted therapy for cancer using pH-responsive nanocarrier systems.pdf. Life sciences. Available at: http://www.sciencedirect.com/science/article/pii/S0024320512000586 [Accessed May 7, 2016].

Manuscript, A., 2009. Quantum dots and nanoparticles for photodynamic and radiation. , 60(15), pp.1600-1614.

Masood, F., 2016. Polymeric nanoparticles for targeted drug delivery system for cancer therapy. Materials Science and Engineering $C, \quad 60$, pp.569-578. Available at: http://dx.doi.org/10.1016/j.msec.2015.11.067.

Mastrobattista, E., Koning, G.A. \& Storm, G., 1999. Immunoliposomes for the targeted delivery of antitumor drugs. Advanced Drug Delivery Reviews, 40(1-2), pp.103-127.

Maya, S. et al., 2013. Smart stimuli sensitive nanogels in cancer drug delivery and imaging: A review. Current Pharmaceutical Design, 19(41), pp.7203-7218. Available at: http://www.scopus.com/inward/record.url?eid=2-s2.0-

84890932990\&partnerID=40\&md5=4fc04c35b444c77bab9c2ae209019cfe [Accessed May 7, 2016].

Mayer, L.D., 2006. Ratiometric dosing of anticancer drug combinations: Controlling drug ratios after systemic administration regulates therapeutic activity in tumor-bearing mice. Molecular Cancer Therapeutics, 5(7), pp.1854-1863. Available at: http://mct.aacrjournals.org/cgi/doi/10.1158/15357163.MCT-06-0118.

McAllister, C., Ramachandran, R. \& Ruetsch, S., 2007. Impact of Nanotechnology on Hair Attributes. ACS Nano, 3(1), pp.1-7. Available at: http://pubs.acs.org/doi/pdf/10.1021/nn900002m [Accessed May 7, 2016].

Meng, H. et al., 2015. Use of a Lipid-Coated Mesoporous Silica Nanoparticle Platform for Synergistic Gemcitabine and Paclitaxel Delivery to Human Pancreatic Cancer in Mice. ACS Nano, 9(4), pp.3540-3557. Available at: http://pubs.acs.org/doi/abs/10.1021/acsnano.5b00510.

Meyers, J. \& Cheng, Y., 2015. Peptide-Targeted Gold Nanoparticles for Photodynamic Therapy of Brain Cancer. Particle \& Particle ... Available at: http://onlinelibrary.wiley.com/doi/10.1002/ppsc.201400119/full [Accessed June 5, 2016].

Mikkelsen, R.B. \& Wardman, P., 2003. Biological chemistry of reactive oxygen and nitrogen and radiation-induced signal transduction mechanisms. Oncogene, 22(37), pp.5734-54. Available at: http://www.ncbi.nlm.nih.gov/pubmed/12947383 [Accessed May 7, 2016].

Miyoshi, N. et al., 2016. Combination of Sonodynamic and Photodynamic Therapy against Cancer Would Be Effective through Using a Regulated Size of Nanoparticles. Nanoscience and Nanoengineering, 4(1), pp.1-11.

Morgan, M.T. et al., 2006. Dendrimer-encapsulated camptothecins: Increased solubility, cellular uptake, and cellular retention affords enhanced anticancer activity in vitro. Cancer Research, 66(24), pp.11913-11921.

Moussa, H.G., Martins, A.M. \& Husseini, G.A., 2015. Review on Triggered Liposomal Drug Delivery with a Focus on Ultrasound,

Nel, A., 2007. Toxic Potential of Materials. Science, 311(5726), pp.622-627. Available at: http://science.sciencemag.org/content/311/5761/622.short [Accessed May 7, 2016].

Newman, C.M.H. \& Bettinger, T., 2007. Gene therapy progress and prospects: ultrasound for gene transfer. Gene therapy, 14(September 2006), pp.465-475. Available at: http://www.nature.com/gt/journal/v14/n6/abs/3302925a.html [Accessed June 6, 2016].

Nikitaki, Z., Mavragani, I. \& Laskaratou, D., 2016. Systemic mechanisms and effects of ionizing radiation: A new "old"paradigm of how the bystanders and distant can become the players. Seminars in cancer ... Available at: http://www.sciencedirect.com/science/article/pii/S1044579X16300049 [Accessed June 6, 2016]. 
Oliveira, C.S. et al., 2011. Major determinants of photoinduced cell death: Subcellular localization versus photosensitization efficiency. Free Radical Biology and Medicine, 51(4), pp.824-833. Available at: http://www.sciencedirect.com/science/article/pii/S089158491100325X [Accessed June 5, 2016].

Omar, G.S., Wilson, M. \& Nair, S.P., 2008. Lethal photosensitization of wound-associated microbes using indocyanine green and near-infrared light. BMC Microbiology, 8(1), p.111. Available at: http://bmcmicrobiol.biomedcentral.com/articles/10.1186/1471-2180-8-111 [Accessed November 20, 2017].

Osminkina, L.A. et al., 2012. Photoluminescent biocompatible silicon nanoparticles for cancer theranostic applications. Journal of Biophotonics, 5(7), pp.529-535. Available at: http://doi.wiley.com/10.1002/jbio.201100112 [Accessed November 6, 2017].

Ozdemir, T. et al., 2017. Generation of Singlet Oxygen by Persistent Luminescent NanoparticlePhotosensitizer Conjugates: A Proof of Principle for Photodynamic Therapy without Light. ChemPhotoChem, 1(5), pp.183-187. Available at: http://doi.wiley.com/10.1002/cptc.201600049 [Accessed November 6, 2017].

Pan, Y. et al., 2009. Gold nanoparticles of diameter $1.4 \mathrm{~nm}$ trigger necrosis by oxidative stress and mitochondrial damage. Small, 5(18), pp.2067-2076.

Park, E.-J. et al., 2008. Oxidative stress and apoptosis induced by titanium dioxide nanoparticles in cultured BEAS-2B cells. Toxicology Letters, 180(3), pp.222-229. Available at: http://linkinghub.elsevier.com/retrieve/pii/S0378427408010552 [Accessed November 6, 2017].

Park, J.W. et al., 1997. Immunoliposomes for Cancer Treatment. Advances in Pharmacology, 40(C), pp.399-435.

Patra, H.K. et al., 2007. Cell selective response to gold nanoparticles. Nanomedicine: Nanotechnology, Biology, and Medicine, 3(2), pp.111-119. Available at: http://www.sciencedirect.com/science/article/pii/S1549963407000536 [Accessed May 7, 2016].

Plastaras, J. et al., 2007. Cell cycle-dependent and schedule-dependent antitumor effects of sorafenib combined with radiation. Cancer research. Available at: http://cancerres.aacrjournals.org/content/67/19/9443.short [Accessed June 6, 2016].

Putnam, D., 2008. Drug delivery: the heart of the matter. Nature materials, 7(11), pp.836-7. Available at: http://www.nature.com/doifinder/10.1038/nmat2309 [Accessed November 6, 2017].

Qian, C. et al., 2017. Conjugated polymer nanomaterials for theranostics. Acta Pharmacologica Sinica, 38(6), pp.764-781. Available at: http://www.nature.com/doifinder/10.1038/aps.2017.42.

Radhakrishnan, K. et al., 2015. Mesoporous silica-chondroitin sulphate hybrid nanoparticles for targeted and bio-responsive drug delivery. New J. Chem., 39(3), pp.1754-1760. Available at: http://xlink.rsc.org/?DOI=C4NJ01430H [Accessed November 6, 2017].

Rasmussen, J.W. et al., 2010. Zinc oxide nanoparticles for selective destruction of tumor cells and potential for drug delivery applications. Expert opinion on drug delivery, 7(9), pp.1063-77. Available at: http://www.tandfonline.com/doi/full/10.1517/17425247.2010.502560 [Accessed November 6, 2017].

Régnard, P. \& Bräuer-Krisch, E., 2008. Enhancement of survival of 9L gliosarcoma bearing rats following intracerebral delivery of drugs in combination with microbeam radiation therapy. European journal of ... Available at: http://www.sciencedirect.com/science/article/pii/S0720048X08003501 [Accessed June 6, 2016].

Rehman, M.U. et al., 2015. Comparison of free radicals formation induced by cold atmospheric plasma, ultrasound, and ionizing radiation. Archives of Biochemistry and Biophysics, pp.1-7. Available at: http://linkinghub.elsevier.com/retrieve/pii/S0003986116301151.

Rengeng, L. et al., 2017. Sonodynamic therapy, a treatment developing from Photodynamic therapy. Photodiagnosis and Photodynamic Therapy. Available at: http://linkinghub.elsevier.com/retrieve/pii/S1572100016302320.

Ricci-Júnior, E. \& Marchetti, J., 2006. Zinc (II) phthalocyanine loaded PLGA nanoparticles for photodynamic therapy use. International journal of pharmaceutics. Available at: http://www.sciencedirect.com/science/article/pii/S0378517305008094 [Accessed June 5, 2016].

Roblero-Bartolon, G.V. \& Ramon-Gallegos, E., 2015. [Use of nanoparticles (NP) in photodynamic therapy (PDT) against cancer]. Gaceta medica de Mexico, 151(1), pp.85-98.

Roh, Y.J. et al., 2017. Photodynamic Therapy Using Photosensitizer-Encapsulated Polymeric 
Nanoparticle to Overcome ATP-Binding Cassette Transporter Subfamily G2 Function in Pancreatic Cancer. Molecular Cancer Therapeutics, 16(8), pp.1487-1496. Available at: http://www.ncbi.nlm.nih.gov/pubmed/28416605 [Accessed November 3, 2017].

Rosa, S. et al., 2017. Biological mechanisms of gold nanoparticle radiosensitization. Cancer Nanotechnology, $\quad 8(1), \quad$ p.2. Available ht: $1 /$ cancernano.springeropen.com/articles/10.1186/s12645-017-0026-0.

Rothkamm, K. \& Löbrich, M., 2003. Evidence for a lack of DNA double-strand break repair in human cells exposed to very low x-ray doses. Proceedings of the National Academy of Sciences of the United States of America, 100(9), pp.5057-62. Available at: http://www.pubmedcentral.nih.gov/articlerender.fcgi?artid=154297\&tool=pmcentrez\&rendertype $=$ abstract [Accessed May 7, 2016].

Saad, M., Garbuzenko, O.B. \& Minko, T., 2008. Co-delivery of siRNA and an anticancer drug for treatment of multidrug-resistant cancer. Nanomedicine (London, England), 3(6), pp.761-776.

Salonen, J. et al., 2005. Mesoporous silicon microparticles for oral drug delivery: Loading and release of five model drugs. Journal of Controlled Release, 108(2-3), pp.362-374. Available at: http://www.sciencedirect.com/science/article/pii/S0168365905003974 [Accessed November 6, 2017].

Sasaki, Y. et al., 2010. Polysaccharide nanogel-cyclodextrin system as an artificial chaperone for in vitro protein synthesis of green fluorescent protein. Polymer Journal, 42(10), pp.823-828. Available at: http://dx.doi.org/10.1038/pj.2010.73 [Accessed May 7, 2016].

Secret, E. et al., 2014. Two-photon excitation of porphyrin-functionalized porous silicon nanoparticles for photodynamic therapy. Advanced Materials, 26(45), pp.7643-7648. Available at: http://doi.wiley.com/10.1002/adma.201403415 [Accessed November 6, 2017].

Sharma, A.K. et al., 2017. Dendrimer nanoarchitectures for cancer diagnosis and anticancer drug delivery. Drug Discovery Today, 22(2), pp.314-326. Available at: http://dx.doi.org/10.1016/j.drudis.2016.09.013.

Sharma, H. et al., 2015. Metal nanoparticles: A theranostic nanotool against cancer. Drug Discovery Today, 20(9), pp.1143-1151. Available at: http://dx.doi.org/10.1016/j.drudis.2015.05.009 [Accessed November 6, 2017].

Shukla, R. et al., 2005. Biocompatibility of gold nanoparticles and their endocytotic fate inside the cellular compartment: A microscopic overview. Langmuir, 21(23), pp.10644-10654. Available at: https://pubs.acs.org/doi/full/10.1021/la0513712?src=recsys [Accessed May 7, 2016].

Simone, C., II, S. \& Heinzerling, J., 2015. Novel radiotherapy approaches for lung cancer: combining radiation therapy with targeted and immunotherapies. Translational lung cancer .... Available at: http://www.ncbi.nlm.nih.gov/pmc/articles/PMC4630515/ [Accessed June 6, 2016].

Singh, N. et al., 2013. Nanogel Based Artificial Chaperone Technology : an Overview. American Journal of Advanced Drug Delivery. Available at: http://ojadd.com/index.php/AJADD/article/view/26 [Accessed May 7, 2016].

Sivaram, A.J. et al., 2015. Nanogels for delivery, imaging and therapy. Wiley Interdisciplinary Reviews: Nanomedicine and Nanobiotechnology. Available at: http://onlinelibrary.wiley.com/doi/10.1002/wnan.1328/full [Accessed May 7, 2016].

Song, X. et al., 2015. Photosensitizer-Conjugated Albumin-Polypyrrole Nanoparticles for ImagingGuided in Vivo Photodynamic/Photothermal Therapy. Small, 11(32), pp.3932-3941. Available at: http://doi.wiley.com/10.1002/smll.201500550 [Accessed November 6, 2017].

Soni, G. \& Yadav, K.S., 2016. Nanogels as potential nanomedicine carrier for treatment of cancer: A mini review of the state of the art. Saudi Pharmaceutical Journal, 24(2), pp.133-139. Available at: http://www.sciencedirect.com/science/article/pii/S1319016414000322.

Stojanovic, V. et al., 2016. Potential of porous silicon nanoparticles as an emerging platform for cancer theranostics. J. Mater. Chem. B, 4(44), pp.7050-7059. Available at: http://xlink.rsc.org/?DOI=C6TB01829G.

Stuart, M. a C. et al., 2010. Emerging applications of stimuli-responsive polymer materials. Nature materials, 9(2), pp.101-113. Available at: http://dx.doi.org/10.1038/nmat2614 [Accessed May 7, 2016].

Studer, A.M. et al., 2010. Nanoparticle cytotoxicity depends on intracellular solubility: Comparison of stabilized copper metal and degradable copper oxide nanoparticles. Toxicology Letters, 197(3), pp.169-174. Available at: http://www.sciencedirect.com/science/article/pii/S0378427410015298 
[Accessed November 6, 2017].

Sultana, F. et al., 2013. An overview of nanogel drug delivery system. Journal of Applied Pharmaceutical Science, 3(8 SUPPL). Available at: http://japsonline.com/admin/php/uploads/1060_pdf.pdf [Accessed May 7, 2016].

Sun, C. et al., 2008. Tumor-targeted drug delivery and MRI contrast enhancement by chlorotoxinconjugated iron oxide nanoparticles. Nanomedicine, 3(4), pp.495-505. Available at: http://www.futuremedicine.com/doi/10.2217/17435889.3.4.495 [Accessed November 6, 2017].

Ta, T. \& Porter, T.M., 2013. Thermosensitive liposomes for localized delivery and triggered release of chemotherapy. Journal of Controlled Release, 169(1-2), pp.112-125.

Taghdisi, S.M. et al., 2016. Double targeting and aptamer-assisted controlled release delivery of epirubicin to cancer cells by aptamers-based dendrimer in vitro and in vivo. European Journal of Pharmaceutics and Biopharmaceutics, 102, pp.152-158.

Tailor, R. et al., 2015. Tumors to Megavoltage Radiation Therapy in vivo. , 11(5), pp.1277-1283.

Tang, Y. et al., 2015. An aptamer-targeting photoresponsive drug delivery system using "off-on" graphene oxide wrapped mesoporous silica nanoparticles. Nanoscale, 7(14), pp.6304-6310. Available at: http://xlink.rsc.org/?DOI=C4NR07493A [Accessed November 6, 2017].

Terentyuk, G. et al., 2014. Gold nanorods with a hematoporphyrin-loaded silica shell for dual-modality photodynamic and photothermal treatment of tumors in vivo. Nano Research, 7(3), pp.325-337.

Tian, W. \& Ma, Y., 2013. Theoretical and computational studies of dendrimers as delivery vectors. Chemical Society Reviews, pp.705-727. Available at: http://pubs.rsc.org/en/content/articlehtml/2013/cs/c2cs35306g [Accessed May 7, 2016].

Tila, D. et al., 2015. Functional liposomes in the cancer-targeted drug delivery. Journal of Biomaterials Applications, $\quad 30(1), \quad$ pp.3-16. Available at: http://jba.sagepub.com/cgi/doi/10.1177/0885328215578111.

Tiwle, R., Giri, T. \& Tripathi, D., 2012. An Exhaustive Review on Solubility Enhancement for Hydrophobic Compounds by Possible Applications of Novel Techniques. Trends in Applied .... Available at: http://search.proquest.com/openview/fa7d3cdf7e9e738d72c59ff9970c5aa5/1?pqorigsite $=$ gscholar [Accessed May 7, 2016].

Tolstik, E. et al., 2016. Studies of silicon nanoparticles uptake and biodegradation in cancer cells by Raman spectroscopy. Nanomedicine: Nanotechnology, Biology, and Medicine, 12(7), pp.19311940. Available at: http://www.sciencedirect.com/science/article/pii/S1549963416300326 [Accessed November 6, 2017].

Vannucci, L. et al., 2003. Effects of N-acetyl-glucosamine-coated glycodendrimers as biological modulators in the B16F10 melanoma model in vivo. International journal of oncology, 23(2), pp.285-296. Available at: http://www.spandidos-publications.com/10.3892/ijo.23.2.285 [Accessed November 6, 2017].

Villiers, C.L. et al., 2010. Analysis of the toxicity of gold nano particles on the immune system: Effect on dendritic cell functions. Journal of Nanoparticle Research, 12(1), pp.55-60. Available at: http://link.springer.com/article/10.1007/s11051-009-9692-0 [Accessed May 7, 2016].

Vinogradov, S. V., Bronich, T.K. \& Kabanov, A. V., 2002. Nanosized cationic hydrogels for drug delivery: Preparation, properties and interactions with cells. Advanced Drug Delivery Reviews, $54(1)$, pp.135-147. Available at: http://www.sciencedirect.com/science/article/pii/S0169409X01002459 [Accessed May 7, 2016].

Wang, C.F. et al., 2015. Dual-drug delivery by porous silicon nanoparticles for improved cellular uptake, sustained release, and combination therapy. Acta Biomaterialia, 16(1), pp.206-214. Available at: http://www.sciencedirect.com/science/article/pii/S1742706115000318 [Accessed November 6, 2017].

Wang, H. et al., 2014. Magnetic/NIR-thermally responsive hybrid nanogels for optical temperature sensing, tumor cell imaging and triggered drug release. Nanoscale, 6(21), pp.13001-13011. Available at: http://dx.doi.org/10.1039/C4NR03748K [Accessed May 7, 2016].

Watarai, S. et al., 2014. Application of $\mathrm{pH}$-sensitive fusogenic polymer-modified liposomes for development of mucosal vaccines. Veterinary Immunology and Immunopathology, 158(1-2), pp.62-72.

Whitesides, G.M., 2003. The "right" size in nanobiotechnology. Nature Biotechnology, 21(10), pp.11611165. Available at: http://www.nature.com/doifinder/10.1038/nbt872. 
Wood, A. \& Sehgal, C., 2015. A review of low-intensity ultrasound for cancer therapy. Ultrasound in medicine \& biology. Available at: http://www.sciencedirect.com/science/article/pii/S0301562914007662 [Accessed June 6, 2016].

Wozny, A.S. et al., 2017. Gadolinium-based nanoparticles as sensitizing agents to carbon ions in head and neck tumor cells. Nanomedicine: Nanotechnology, Biology, and Medicine.

Wu, P.H. et al., 2016. Gold Nanoparticles With RGD Peptide in Radiation Therapy Suppress the Invasion Activity of Breast Cancer Cells. International Journal of Radiation Oncology*Biology*Physics, 96(2), p.E574. Available at: http://linkinghub.elsevier.com/retrieve/pii/S0360301616323914.

Xia, B. et al., 2013. Engineered stealth porous silicon nanoparticles via surface encapsulation of bovine serum albumin for prolonging blood circulation in vivo. ACS Applied Materials and Interfaces, 5(22), pp.11718-11724. Available at: http://pubs.acs.org/doi/10.1021/am403380e [Accessed November 6, 2017].

$\mathrm{Xu}, \mathrm{H}$. et al., 2016. Nanoparticles in sonodynamic therapy: state of the art review. $R S C A d v ., 6(56)$, pp.50697-50705. Available at: http://xlink.rsc.org/?DOI=C6RA06862F.

Xu, W. et al., 2015. Smart Porous Silicon Nanoparticles with Polymeric Coatings for Sequential Combination Therapy. Molecular Pharmaceutics, 12(11), pp.4038-4047. Available at: http://pubs.acs.org/doi/abs/10.1021/acs.molpharmaceut.5b00473 [Accessed November 6, 2017].

Yang, G. et al., 2017. Hollow $\mathrm{MnO} 2$ as a tumor-microenvironment-responsive biodegradable nanoplatform for combination therapy favoring antitumor immune responses. Nature Communications, $8(1)$, p.902. Available at: http://www.nature.com/articles/s41467-017-01050-0 [Accessed November 6, 2017].

Yang, J. et al., 2014. Mesoporous Silica-Coated Plasmonic Nanostructures for Surface-Enhanced Raman Scattering Detection and Photothermal Therapy. Advanced Healthcare Materials, 3(10), pp.1620 1628. Available at: http://doi.wiley.com/10.1002/adhm.201400053 [Accessed November 6, 2017].

Yang, W. et al., 2009. Targeting cancer cells with biotin-dendrimer conjugates. European Journal of Medicinal Chemistry, 44(2), pp.862-868. Available at: http://www.sciencedirect.com/science/article/pii/S0223523408002195 [Accessed November 6, 2017].

Yatoo, M.I., 2014. Nanotechnology Based Drug Delivery at Cellular Level: a Review. Journal of Animal Science Advances, 4(2), pp.705-709. Available at: http://www.scopemed.org/?mno=37359.

Yin, T. et al., 2017. In vivo high-efficiency targeted photodynamic therapy of ultra-small Fe3O4@polymer-NPO/PEG-Glc@Ce6 nanoprobes based on small size effect. NPG Asia Materials, 9(5), p.e383. Available at: http://www.nature.com/doifinder/10.1038/am.2017.68 [Accessed November 6, 2017].

$\mathrm{Yu}$, J. et al., 2016. Internalized compartments encapsulated nanogels for targeted drug delivery. Nanoscale, pp.1-3. Available at: http://xlink.rsc.org/?DOI=C5NR08895J [Accessed May 7, 2016].

Yu, T., Wang, Z. \& Mason, T.J., 2004. A review of research into the uses of low level ultrasound in cancer therapy. Ultrasonics Sonochemistry, 11(2), pp.95-103.

Yuba, E. et al., 2010. pH-Sensitive fusogenic polymer-modified liposomes as a carrier of antigenic proteins for activation of cellular immunity. Biomaterials, 31(5), pp.943-951. Available at: http://www.sciencedirect.com/science/article/pii/S0142961209010813 [Accessed November 6, 2017].

Zaimy, M.A. et al., 2017. New methods in the diagnosis of cancer and gene therapy of cancer based on nanoparticles. Cancer Gene Therapy, 24(6), pp.233-243. Available at: http://www.nature.com/doifinder/10.1038/cgt.2017.16.

Zalipsky, S. et al., 1996. Long-circulating, polyethylene glycol-grafted immunoliposomes. In Journal of Controlled Release. pp. 153-161.

Zhao, D., 1998. Triblock Copolymer Syntheses of Mesoporous Silica with Periodic 50\&amp;nbsp;to 300\&amp;nbsp;Angstrom Pores. Science, 279(5350), pp.548-552. Available at: http://www.ncbi.nlm.nih.gov/pubmed/9438845 [Accessed November 6, 2017].

Zhao, G. \& Leticia Rodriguez, B., 2012. Molecular targeting of liposomal nanoparticles to tumor microenvironment. International Journal of Nanomedicine, 8, pp.61-71.

Zhou, J. et al., 2010. Dual-modality in vivo imaging using rare-earth nanocrystals with near-infrared to near-infrared (NIR-to-NIR) upconversion luminescence and magnetic resonance properties. Biomaterials,

31(12),

pp.3287-3295

Available

at: 
http://www.sciencedirect.com/science/article/pii/S0142961210000566 [Accessed November 20, 2017].

Zununi Vahed, S. et al., 2017. Liposome-based drug co-delivery systems in cancer cells. Materials Science and Engineering $C$, 71, pp.1327-1341. Available at: http://www.sciencedirect.com/science/article/pii/S0928493116322871 [Accessed November 6, 2017]. 